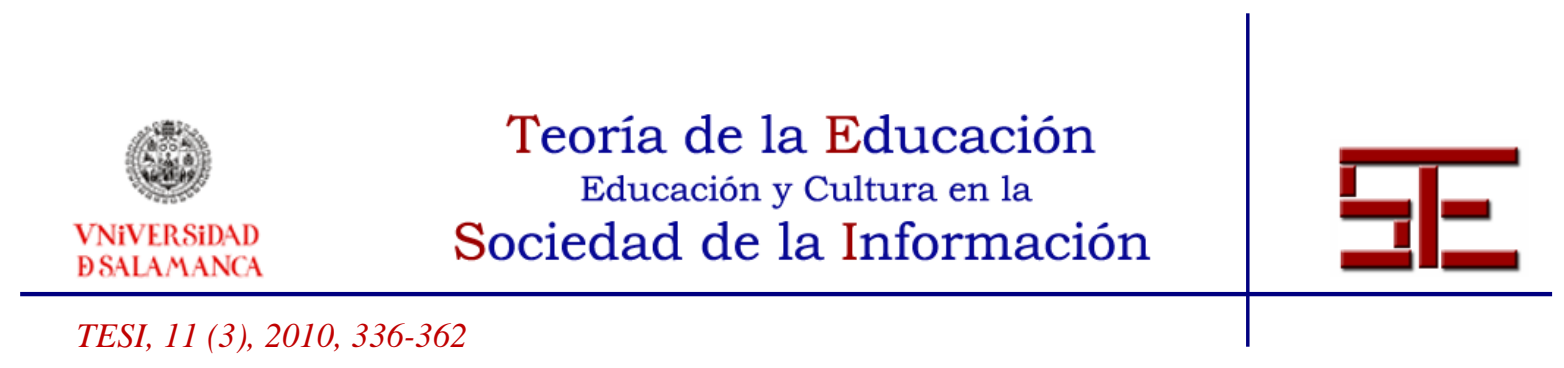

\title{
IDENTIFICACIÓN DEL DOMINIO DE COMPETENCIAS DIGITALES EN EL ALUMNADO DEL GRADO DE MAGISTERIO
}

Resumen: Las Tecnologías de la información y comunicación (TIC) se convierten en la sociedad de la Información en un agente de cambio. En este contexto las TIC deben transformarse en herramientas pedagógicas al servicio del profesor con el fin de lograr una educación de calidad.

Conscientes de la importancia de que los profesores dominen las competencias digitales, hemos realizado un estudio sobre el dominio de las tecnologías de la información y la comunicación (TIC) que poseen los estudiantes de tercer curso de la titulación de Magisterio de la Universidad de Vigo. Para ello se han tenido en cuenta los conocimientos adquiridos, la frecuencia en la utilización de determinadas herramientas, su nivel de dominio en cuatro ámbitos de conocimiento: Alfabetización tecnológica, Instrumentos de trabajo intelectual, Tratamiento y difusión de la información y como Herramientas de comunicación. Así como sus motivaciones e intereses y los obstáculos encontrados en su desarrollo con el fin de establecer propuestas para la formación inicial.

Podemos concluir que, en general, el alumnado no tiene una formación específica sobre el funcionamiento de los ordenadores. Sobre el grado de conocimiento de las diferentes destrezas el alumno conoce las más básicas y las que utilizan habitualmente (abrir o bajar un archivo, crear o imprimir un documento, instalar un programa o enviar un email). Utilizan habitualmente el correo como una herramienta de trabajo, mientras que la mensajería y las redes sociales las utilizan más en tiempos de ocio.

Sus actitudes hacia las TIC son muy positivas y sus motivaciones se centran fundamentalmente en que las tecnologías son útiles para mejorar su aprendizaje y para su futuro laboral.

Palabras clave: TIC; competencias digitales; tecnología educativa; educación superior; formación del profesorado

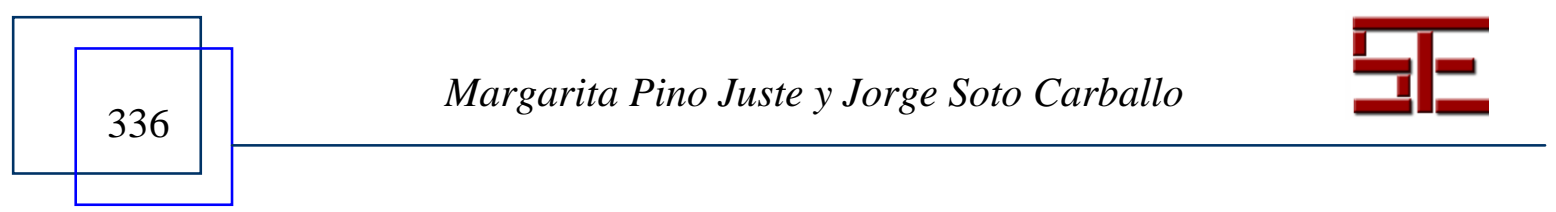




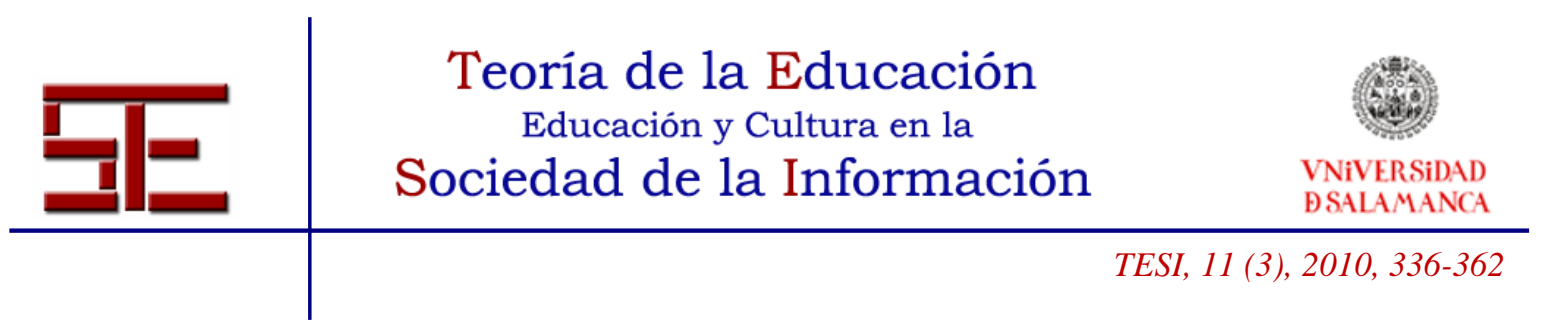

\title{
IDENTIFICATION OF DIGITAL COMPETENCES SKILLS IN TEACHER TRAINING DEGREE STUDENTS
}

\begin{abstract}
:
The Technologies of Information and Communication (ICT) become in the information society a change agent. In this context, ICTs should become teaching tools in order to help the teacher to achieve quality education.

Being aware of the importance of the teachers' mastery of the digital skills, we have conducted a study about the mastery of the ICTs that the students in the third year of the degree of teacher training of the University of Vigo have. In order to do this we have taken into account the knowledge acquired, the frequency of use of certain tools, their level of proficiency in four areas of knowledge: technological literacy, intellectual working tools, processing and dissemination of information and as communication tools. As well as their motivations, interests and obstacles found in their development in order to develop proposals for initial training.

We can conclude that, in general, students do not have a specific training on the use of computers. About the degree of knowledge in the different skills, the students know the most basic and commonly used (open or download a file, create or print a document, install a program or send an e-mail). They usually use the mail as a working tool, while the messaging and social networks are more used for leisure time.

Their attitudes towards ICTs are very positive and their motivations are focused essentially on the technologies which are useful for improving their learning and for their professional future.
\end{abstract}

Keywords: ICT; digital competences; educational technology; higher education; teacher training.

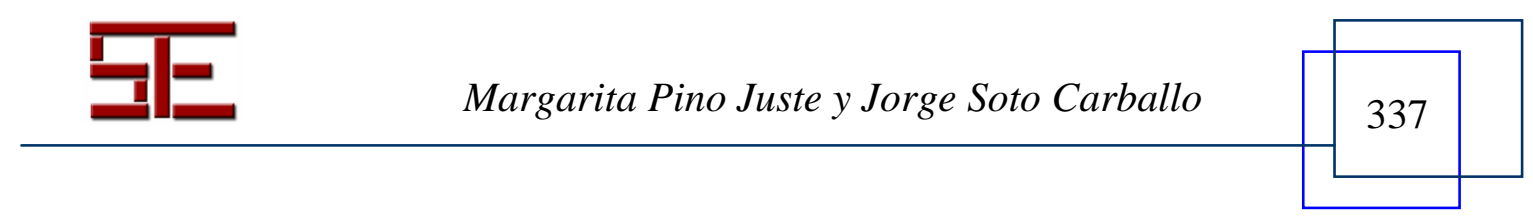




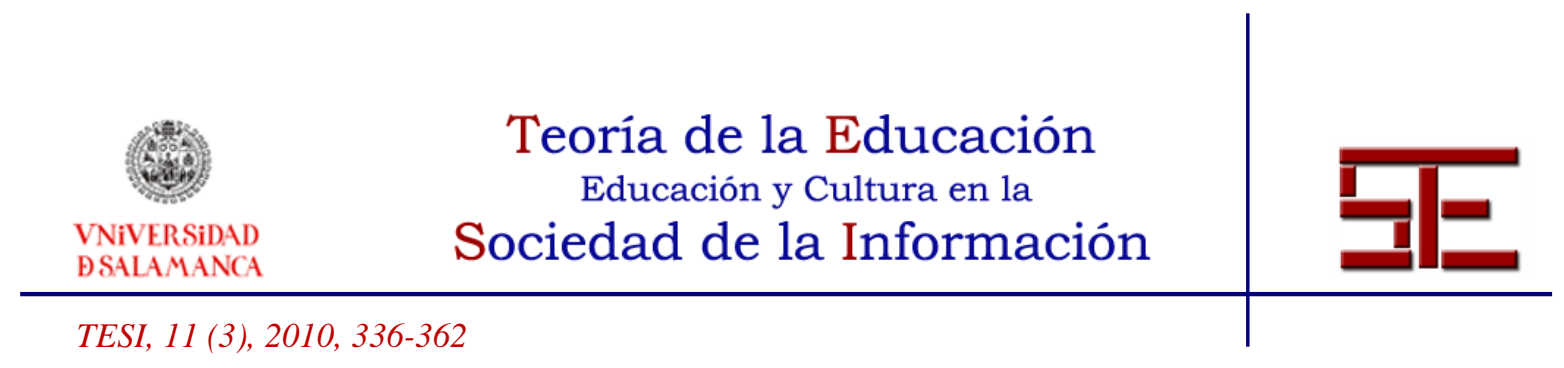

\title{
IDENTIFICACIÓN DEL DOMINIO DE COMPETENCIAS DIGITALES EN EL ALUMNADO DEL GRADO DE MAGISTERIO
}

Fecha de recepción: 16/04/2010; fecha de aceptación: 05/09/2010; fecha de publicación: 30/11/2010

\author{
Margarita Pino Juste \\ mpino@uvigo.es \\ Universidad de Vigo \\ Jorge Soto Carballo \\ hesoto@uvigo.es \\ Universidad de Vigo
}

\section{1.- CONTEXTO E IMPORTANCIA DE LA INVESTIGACIÓN}

La creciente importancia y la atención prestada a las TIC han ido creciendo paralelamente, desde hace años, al potencial de las nuevas tecnologías con el fin de mejorar el proceso de enseñanza-aprendizaje en la escuela (Álvarez Castillo, 2001; Clarke, 2004).

De hecho, McFarlane $(2001,237)$ señala que, desde los estamentos políticos, se describe la visión sobre las TIC como un agente de cambio que se aplica de un modo revolucionario.

Así, durante varias décadas en todos los países del mundo el mayor impulso en el ámbito educativo ha sido la búsqueda de formulas para que los profesores se convenzan de que las TIC deben ser parte integral de su estrategia de enseñanza. Han sido impulsadas diferentes estrategias como la difusión de buenas prácticas, la inversión en infraestructuras y equipamiento y, en los últimos años, la implementación de programas de formación docente destinados a aumentar los niveles de cualificación y fomentar actitudes positivas hacia los ordenadores.

Sin embargo, la mayor parte de la investigación sobre el empleo de las TIC en la escuela indica que los ordenadores no han tenido un impacto tan revolucionario como se esperaba sobre la práctica docente de los profesores ni sobre el aprendizaje de los estudiantes (Knezek y Christensen, 2002, Harrison et al. 2002; Nichol \& Watson, 2003; Reynolds et al. 2003; Selwyn, 1999, 2003; Cuadrado y Fernández, 2003). Tampoco el hecho de dotar a los centros de recursos informáticos ha favorecido la integración de las TIC en la práctica escolar (Urkijo, 2004;

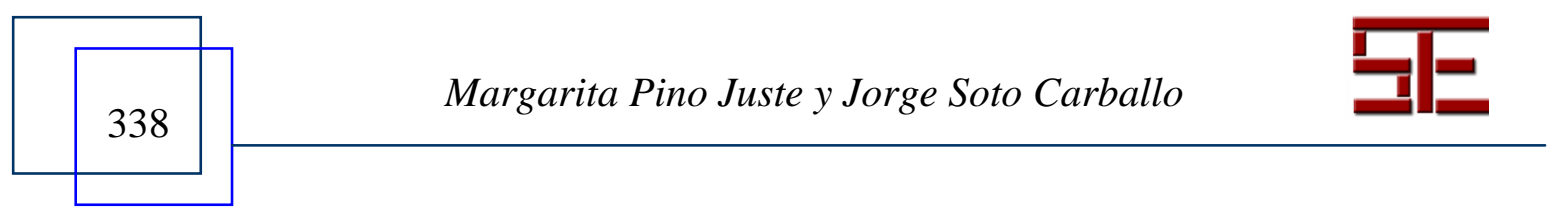




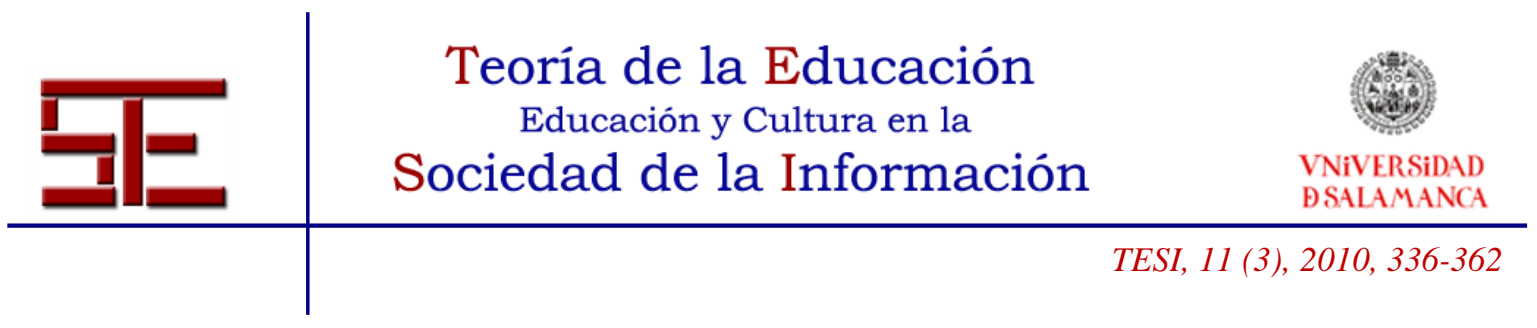

Barquín Ruiz, 2005; Scrimshaw, 2004; Mooij, 2004). Esta situación obliga a investigar cuáles son las causas que impiden que el profesorado utilice las herramientas tecnológicas de las que dispone para mejorar el proceso de enseñanza-aprendizaje.

Ya existen investigaciones que analizan las razones de este fracaso y las condiciones previas que, con bastantes probabilidades, contribuyen a lograr las capacidades necesarias para manejar correctamente las TIC en los procesos de enseñanza-aprendizaje (Cuckle et al. 2000; Fisher, 2000).

Lo que es evidente desde la nueva conformación del sistema educativo, a partir de la aparición de las nuevas tecnologías, es que es indudable que los avances tecnológicos a los que nos enfrentamos hoy en día están condicionando el modelo de sociedad que conocemos (García Aretio, 2007).

Sabemos que el estudiante del futuro tendrá que poseer diferentes competencias básicas para aprender conocimientos nuevos, desaprender conocimientos adquiridos y ya no validos por la trasformaciones del mundo científico y reaprender los nuevos conocimientos que se vayan generando (Cabero y Barroso, $2007,42)$. Este es sin duda un reto que concierne a toda la sociedad.

Pero además debemos tener en cuenta tal como señala Adell (1998) que las nuevas tecnologías de la información y comunicación están transformando el modo en que trabajamos, pero también en el que pasamos nuestro tiempo libre y nos divertimos, nos relacionamos con otras personas $y$, en general, nos transforma como ciudadanos y por extensión a toda la sociedad.

Desde estos argumentos, y con el fin de sacar el máximo rendimiento a las TIC el sistema educativo formal, consideramos pertinente preguntarnos cuál o cuáles son los modelos pedagógicos y las competencias que demanda la sociedad informacional y comunicada, entendida como un nuevo enfoque dentro de la Pedagogía que estudia los procesos de enseñanza-aprendizaje y que tiene a la información y a la comunicación como eje y centro de interés fundamental.

Hablamos, por lo tanto, de identificar las nuevas necesidades formativas básicas, genéricas y específicas que doten de solvencia competencial al individuo, entendida dicha competencia como "el conjunto de conocimientos, destrezas y actitudes que ha de ser capaz de movilizar una persona, de forma integrada, para actuar eficazmente ante las demandas de un determinado contexto" (Mérida, 2006, 676).

En este punto es preciso acercarnos al constructo de competencia ya que tal como argumentan Prins, Nadolski, Berlanga, Drachsler, Hummel y Koper (2008) este concepto aporta las fortalezas necesarias para llevar a cabo, entre otras, las oportunas recomendaciones personales apropiadas a cada individuo con el fin de

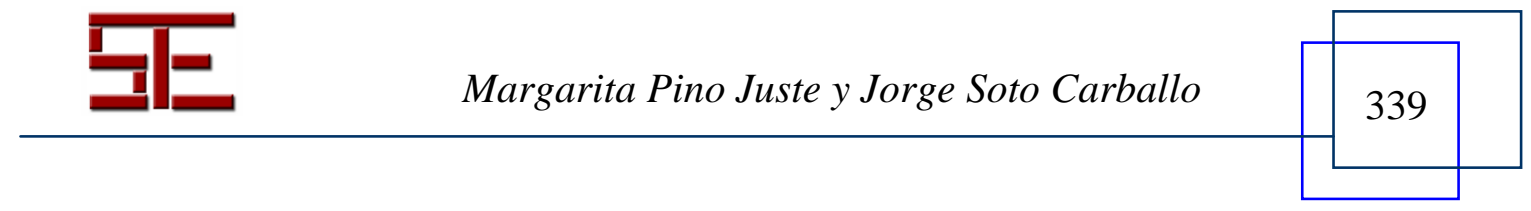




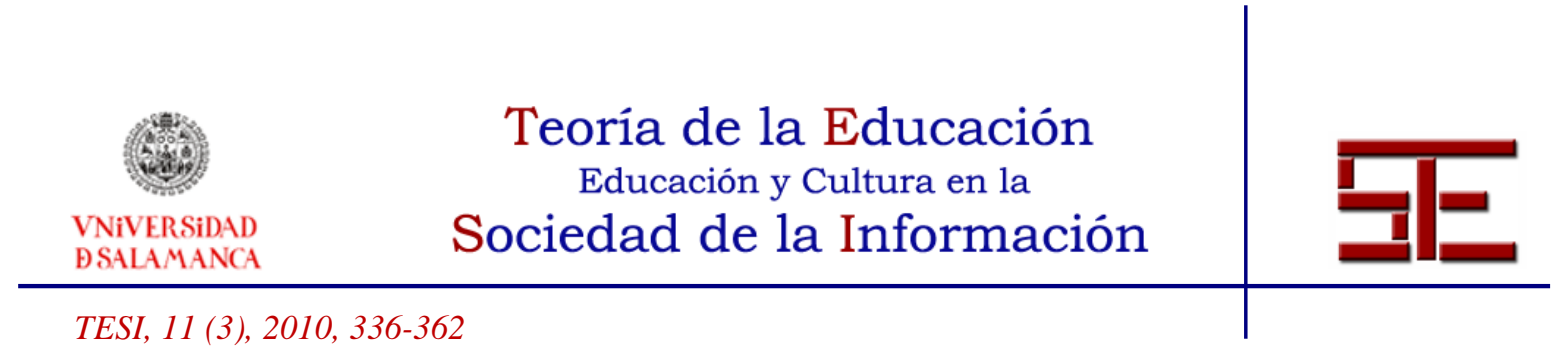

realizar una selección adecuada de las actividades de aprendizaje, para determinar niveles de competencias individualizadas, para el diseño de tareas de aprendizaje o para acreditar un nuevo conocimiento. En este contexto los autores proponen un nuevo concepto "Learning and performance situation" (LP-situation).

Estos nuevos modelos, generados a partir de las nuevas relaciones que se producen en la Sociedad del Conocimiento representan nuevos retos que permitan transformar la información en conocimiento y el conocimiento en educación.

Así, cuando hablamos de educación mediada por la tecnología informática, desde la escuela no podemos olvidar la necesidad de proponer claves para desarrollar estrategias imaginativas y creativas que entronquen con los componentes cognitivos y cognitivo lingüísticos (pensar, comprender, decidir, buscar información, comunicarse oralmente y por escrito, utilizar las nuevas y tecnologías), con los elementos emocionales y de equilibrio personal (desarrollar un proyecto de vida...) con los elementos de relación interpersonal (cooperar, trabajar en equipo...) o con los de actuación e de inserción social (comunicarse con miembros de otras culturas, compartir...) ( Coll, 2007).

Se trata, en definitiva, de generar pautas de intervención adecuadas de cara a producir correlaciones óptimas en el tratamiento de la información por parte de los usuarios que comporten la asunción de nuevos aprendizajes "clave" para la formación básica del alumnado.

Es por ello que puede resultar relevante proponer claves de actuación basadas en la adaptación de la comunicación y la información a los alumnos para crear fortalezas que potencien valores humanos $\mathrm{y}$ mediáticos que conviertan el proceso comunicativo e informativo en un continuo feedback, en una interacción beneficiosa con lo virtual. Esto conlleva un esfuerzo por atender a la diversidad del alumnado con el fin de propiciar el desarrollo de competencias.

Se evidencian las fortalezas de las TIC como agentes generadores de procesos creativos y como activador de las secuencias de aprendizaje. Asimismo, implica innovar a través de propuestas creativas generando contenidos multimedia imaginativos que produzcan satisfacción y aprendizaje significativo. Cobra fuerza aquí las aportaciones de algunos autores al considerar las TIC como herramientas con potencial cognitivo o mindtools (Jonassen, 2006); es decir, como instrumentos que permiten diferentes representaciones del conocimiento a la vez que facilita la reflexión, interiorizándolo significativamente (Coll, Mauri y Onrubia, 2008). Por ello se hace necesario formar e informar sobre los mejores medios de innovación tecnológica basados en estándares de calidad (Aguado Román, 2008) para desarrollar en los usuarios competencias informacionales.

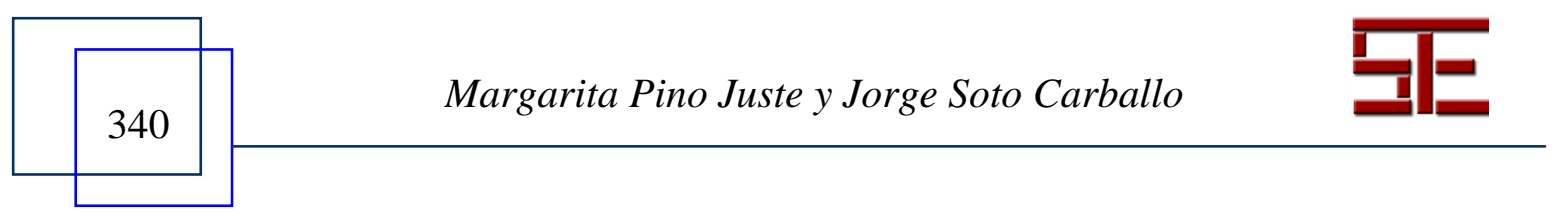




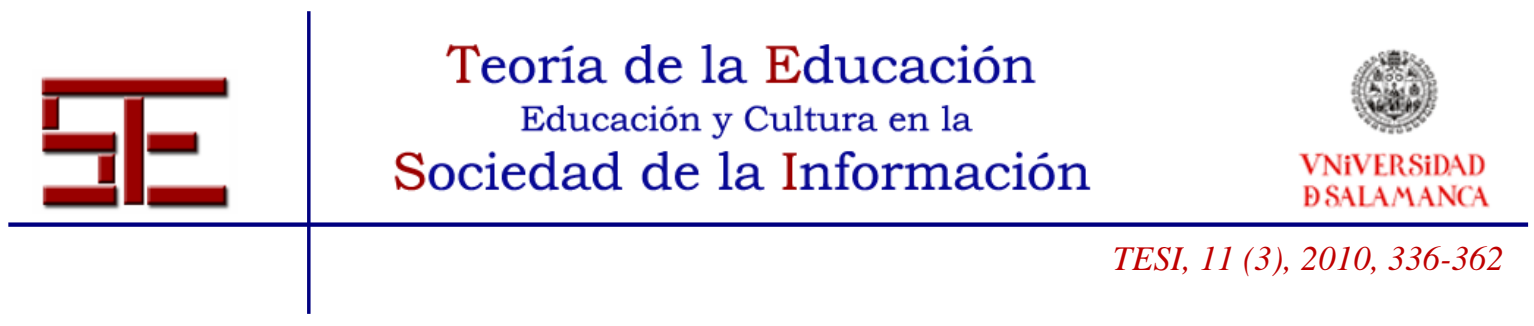

Todo ello exige que durante la formación inicial del profesorado se consiga un nivel básico de destrezas en las competencias digitales que faciliten que el futuro maestro pueda desempeñar su labor con independencia y autonomía.

Tenemos, tal como señala Touriñán (2004), ante nosotros un reto fundamental: lograr una educación de calidad derivada de la sociedad de la información en la que las nuevas tecnologías de la información y las comunicaciones se convierten en herramientas pedagógicas al servicio del profesor.

Conscientes, por lo tanto, de la importancia de este reto, hemos llevado a cabo un estudio que pretende analizar cuáles han sido las competencias digitales adquiridas por el alumnado del grado de magisterio durante su formación inicial. Para ello se han tenido en cuenta los conocimientos adquiridos, la frecuencia en la utilización de determinadas herramientas, su nivel de dominio así como sus motivaciones y los obstáculos encontrados en su desarrollo con el fin de establecer propuestas para la formación inicial.

\section{2.- ESTUDIO}

\section{1.- Participantes}

El presente estudio se realizó con el alumnado de tercero de Magisterio de las especialidades de educación infantil (87 alumnos), primaria (70 alumnos), física (31 alumnos) y musical (31 alumnos) de la Facultad de Educación de la Universidad de Vigo en el Campus de Pontevedra y los profesores que imparten clase en estas titulaciones y emplean la plataforma digital de la Universidad de forma habitual en su docencia.

Por lo tanto, esta investigación ha sido desarrollada con una muestra de 219 estudiantes que han finalizado en el curso 2008-09 su carrera de Magisterio y un total diez profesores de las diferentes especialidades. Solamente seis de ellos participaron en los grupos de discusión, tres en cada grupo.

Con respecto al género del alumnado el 75,3 \% son mujeres y el $24,7 \%$ son hombres. Existe una ligera mayoría de hombres en educación musical $(55 \%)$ aumentando en educación física (71\%). Sin embargo, en educación primaria (56\%) $\mathrm{y}$, sobre todo en educación infantil (86\%) existe una abrumadora mayoría de mujeres.

Sobre la edad, la media es de 21, 33 años siendo la mínima 19 y la máxima 35. La moda es 20.

Con respecto al nivel de estudios de los padres, nos sorprende que aún en porcentajes muy pequeños, haya padres que no tengan estudios y que solamente el

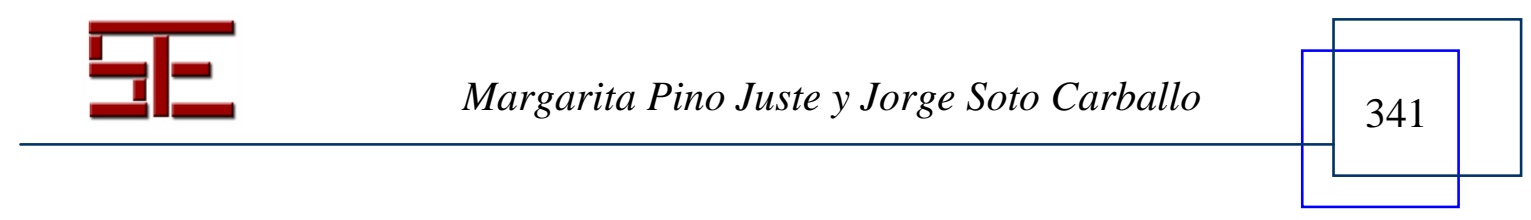




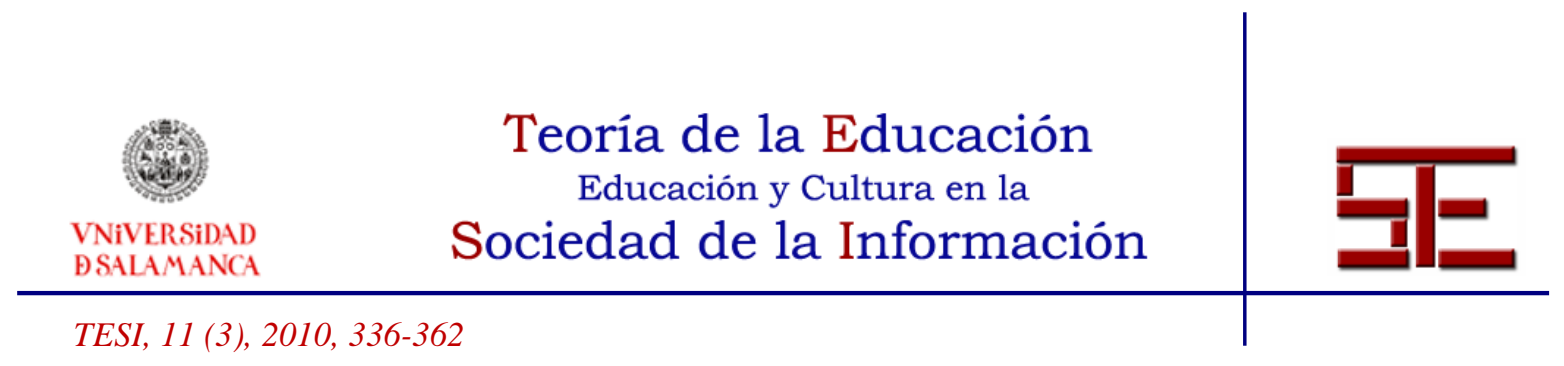

$14 \%$ de los padres tengan estudios universitarios. Asimismo llama la atención que el porcentaje de madres universitarias sea mayor, aunque no de forma significativa $(16 \%)$.

\section{2.- Procedimiento}

La investigación se plantea como un estudio de campo de tipo exploratorio descriptivo definido por Hernández Sampieri, Fernández Collado y Baptista Lucio (2003) como aquel que permite no sólo observar sino recolectar los datos tal y como se dan en su contexto natural para después analizarlos.

El tipo de muestreo empleado es de tipo probabilístico disponible accidental, dado que el fin del estudio no es generalizar los resultados, sino comprender la realidad del grupo clase y establecer correlaciones entre las distintas variables sometidas a estudio. Por ello, la muestra es entendida como "un grupo de sujetos seleccionados sobre la base de ser accesibles o adecuados" (McMillanm y Shumacher, 2005, 140).

La metodología de investigación combina técnicas cualitativas y cuantitativas lo que permite una mayor riqueza de información.

Como instrumento de recogida de datos cuantitativo se utiliza un cuestionario cerrado que se ha aplicado al alumnado de tercero de magisterio de todas las especialidades (Infantil, primaria, musical y educación física) que se imparten en la Facultad de Ciencias de la Educación y el Deporte de la Universidad de Vigo (Campus de Pontevedra).

La técnica de carácter cualitativo fue el grupo de discusión formado por alumnos y profesores. Cada grupo de discusión estaba formado por cinco alumnos y tres profesores de las diferentes especialidades. Es habitual el uso de los grupos de discusión de forma aislada, práctica que algunos autores justifican en el hecho de que conducen a resultados similares a los obtenidos por otros procedimientos (Ward, Bertrand y Brown, 1991). Sin embargo, en nuestro estudio la hemos utilizado como técnica complementaria ya que el objetivo era la obtención de datos sobre las percepciones, opiniones, actitudes, sentimientos o conductas de los sujetos en relación con el dominio del alumnado de magisterio de las competencias digitales y los obstáculos para su adquisición (Gil Flores, 1992).

Para elaborar el cuestionario se han identificado como competencias básicas las descritas en los diferentes documentos institucionales y se han utilizado cuestionarios ya elaborados que medían, bien en su totalidad o en alguna de sus dimensiones, algunas de las variables estudiadas (Gallego, 2007; Mominó y Sigalés, 2009).

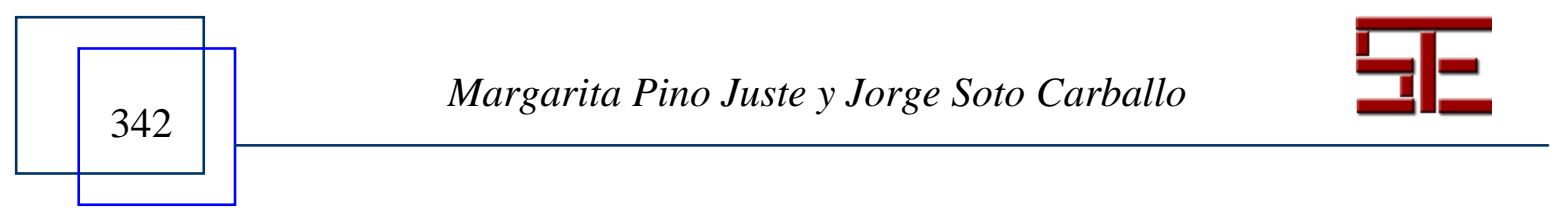




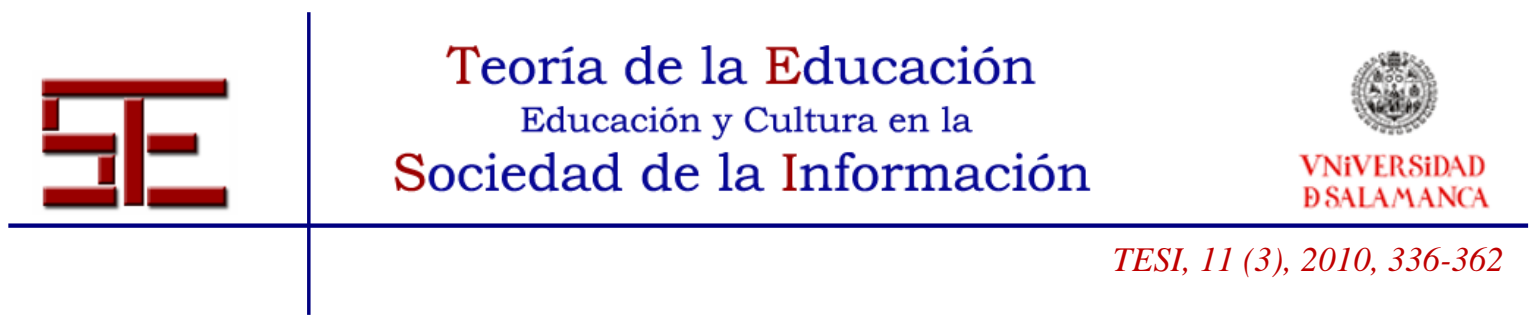

El cuestionario está configurado por 6 ítems con el fin de conocer los datos de identificación del alumnado (sexo, edad, especialidad, nivel de estudios de padre y madre y lugar donde se ha adquirido la formación sobre las TIC), datos sobre conocimiento y experiencia sobre las TIC (25 ítems), datos sobre utilización y frecuencia de las diferentes herramientas (21 ítems), datos sobre motivaciones creencias y actitudes (13 ítem), datos sobre dominio de las TIC y utilización didáctica de los medios (23 ítems), y sobre problemas de utilización de las TIC en el aula (10 ítem). Los ítems se agrupan en dos tipos: de respuesta única y tipo escala Likert de 1 a 5 para medir las actitudes. Las escalas de valoración consisten en un conjunto de ítems bajo la forma de afirmaciones o juicios ante los cuales se solicita la reacción (favorable o desfavorable, positiva o negativa) del alumnado.

Cuando se pasaba el cuestionario se informaba a los estudiantes sobre el objetivo del instrumento y aseguraba el anonimato de las respuestas. El periodo de recogida de los cuestionarios fue la última semana de mayo del año 2009. Los grupos de discusión se desarrollaron durante el mes de julio de 2009.

\section{3.- RESULTADOS}

La mayoría de los alumnos $(43,4 \%)$ no tienen formación específica sobre destrezas en tecnologías de la información y comunicación siendo esta adquirida de forma autodidacta. El 33,3\% la han adquirido durante sus estudios.

\begin{tabular}{|l|c|c|}
\hline & Frecuencia & Porcentaje \\
\hline Sin formación específica & 95 & 43,4 \\
Durante los estudios & 73 & 33,3 \\
En cursos de informática & 28 & 12,8 \\
específicos & 23 & 10,5 \\
Con ayuda de amigos y familia & 219 & 100,0 \\
Total & & \\
\hline
\end{tabular}

Tabla1: Cómo se ha adquirido los conocimientos sobre las TIC

No obstante, como podemos comprobar el grado de dominio y experiencia sobre las TIC es alto, salvo en dos aspectos: el conocimiento de paquetes estadísticos y para diseñar o modificar contenidos en páginas Web, Blogs, etc.

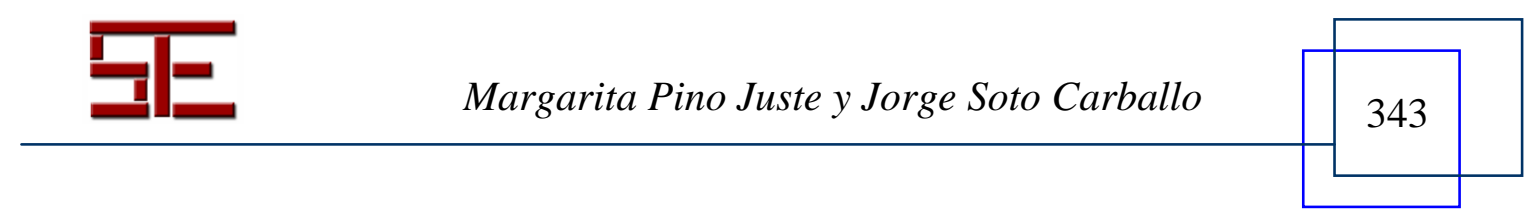




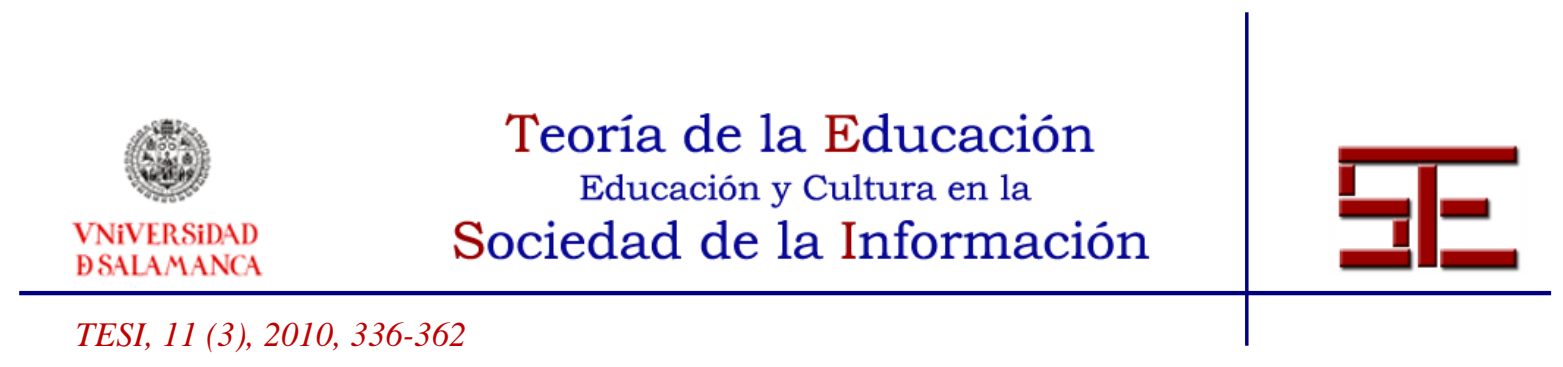

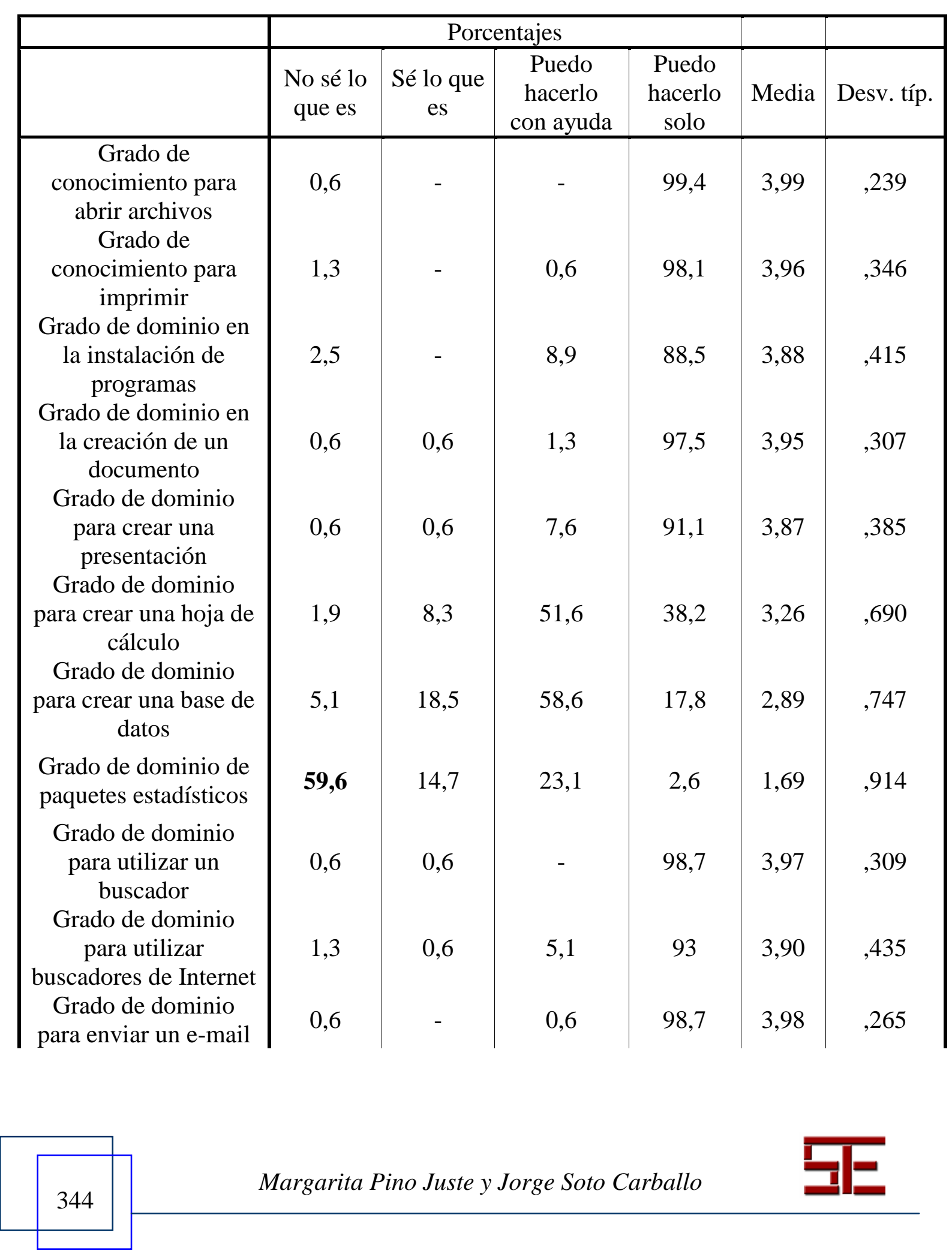




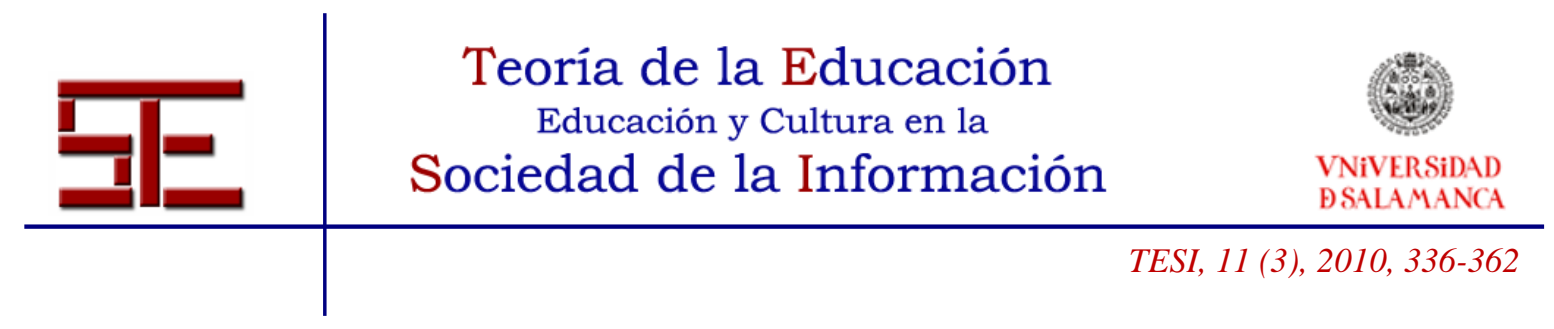

\begin{tabular}{|} 
Grado de dominio \\
para adjuntar un \\
archivo \\
Grado de dominio \\
para utilizar \\
programas de \\
mensajería \\
Grado de dominio \\
para publicar \\
contenidos en Internet \\
Grado de dominio \\
para diseñar o \\
modificar contenidos \\
en páginas Web, Blogs
\end{tabular}

\begin{tabular}{l|r|r|r|r|r|}
0,6 & 1,9 & 1,3 & 96,1 & 3,93 &, 387 \\
0,6 & 1,9 & 1,3 & 96,1 & 3,95 &, 307 \\
1,9 & 7,7 & 19,2 & 71,1 & 3,59 &, 724 \\
3,8 & $\mathbf{3 0 , 6}$ & $\mathbf{3 8 , 2}$ & 27,4 & 2,89 &, 864
\end{tabular}

Tabla 2: Conocimiento y experiencia sobre las TIC

De los datos se infiere que casi todos los alumnos pueden enviar un e-mail sin ayuda, abrir o adjuntar un archivo, imprimir un texto o gráfico, utilizar la instalación de programas, utilizar programas de mensajería o crear un documento así como presentarlo. De hecho, se constata que la mayoría del alumnado lleva más de tres años utilizando Internet y solamente dos personas todavía no utilizan esta herramienta.

Asimismo, se comprueba que todos disponen de correo, salvo dos alumnos. Una importante mayoría utiliza también la mensajería instantánea y las redes sociales y este porcentaje baja cuando preguntamos si disponen de blog propio.

\begin{tabular}{|l|c|c|c|c|}
\hline & Correo & Blog & Mensajería & Redes sociales \\
\hline SI & 99,1 & 60,3 & 95,0 & 95,0 \\
NO & 9 & 39,7 & 5,0 & 5,0 \\
Total & 100,0 & 100,0 & 100,0 & 100,0 \\
\hline
\end{tabular}

Tabla 3: Disponibilidad de recursos

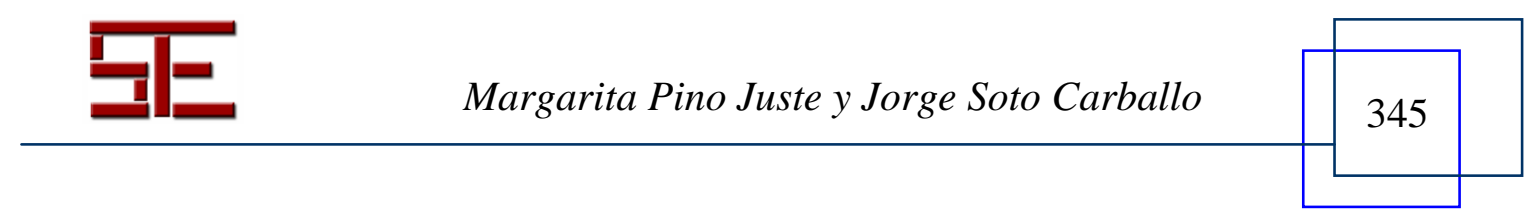




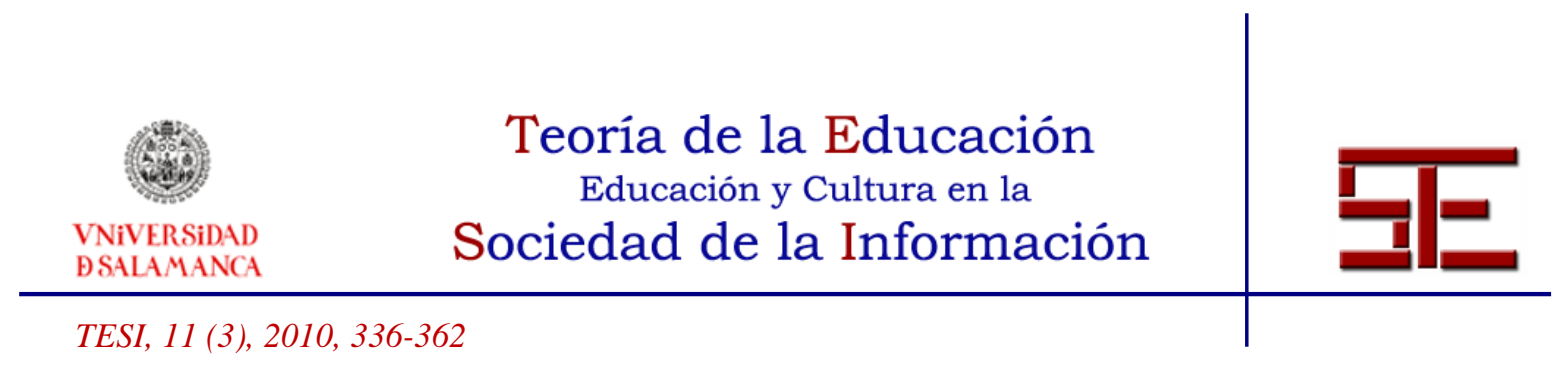

Como podemos observar en los porcentajes siguientes la frecuencia de utilización varía mucho en función de las herramientas.

\begin{tabular}{|c|c|c|c|c|}
\hline & Correo & Blog & Mensajería & Redes Sociales \\
\hline Nunca &, 9 & 41,1 & 2,7 & 2,5 \\
\hline $\begin{array}{c}\text { Menos de una vez } \\
\text { al mes }\end{array}$ & 2,3 & 4,1 & 11,0 & 12,3 \\
\hline 2 o 3 veces la mes & 9,6 & 4,6 & 10,5 & 9,8 \\
\hline $\begin{array}{c}\text { 2 o 3 veces a la } \\
\text { semana }\end{array}$ & 24,2 & 12,3 & 73,1 & 71,5 \\
\hline Cada día & 63,0 & 37,9 & 2,7 & 3,9 \\
\hline Total & 100,0 & 100,0 & 100,0 & 100,0 \\
\hline
\end{tabular}

Tabla 4: Utilización de recursos

Así, en los grupos de discusión hemos averiguado que habitualmente abren su correo porque lo consideran como una herramienta de trabajo y es el medio por el cual se comunican con los profesores; mientras que la mensajería y las redes sociales las utilizan más el fin de semana y en su tiempo libre como herramienta de comunicación con los amigos. Un alumno lo explica así: "Para trabajar utilizas más el correo, para comunicarte con tus amigos y pasar información y hablar de nuestras cosas utilizas la mensajería o las redes y habitualmente las dos al mismo tiempo" (AEI22).

Podemos comprobar en la Tabla adjunta para qué utilizan los alumnos las diferentes herramientas y cuál es la frecuencia.

\begin{tabular}{|c|c|c|c|c|c|c|c|}
\hline & 1 & 2 & 3 & 4 & 5 & Media & Desv. típ. \\
\hline \multirow{4}{*}{$\begin{array}{l}\text { Utilizar un ordenador para } \\
\text { escribir, realizar gráficos, } \\
\text { tablas o dibujos } \\
\text { Utilizar un ordenador para } \\
\text { realizar cálculos } \\
\text { matemáticos } \\
\text { Bajarme música, } \\
\text { películas, juegos u otros } \\
\text { programas } \\
\text { Jugar en línea con otros } \\
\text { amigos }\end{array}$} & 1,4 & 29,2 & 19,6 & 24,2 & 25,1 & 3,43 & 1,19 \\
\hline & 35,2 & 51,1 & 5,9 & 4,6 & 2,7 & 1,88 & 0,913 \\
\hline & 5,5 & 16,0 & 15,1 & 27,4 & 36,1 & 3,73 & 1,25 \\
\hline & 40,6 & 30,1 & 5,9 & 12,8 & 10,0 & 2,21 & 1,36 \\
\hline \multicolumn{7}{|c|}{ Margarita Pino Juste y Jorge Soto Carballo } & \\
\hline
\end{tabular}



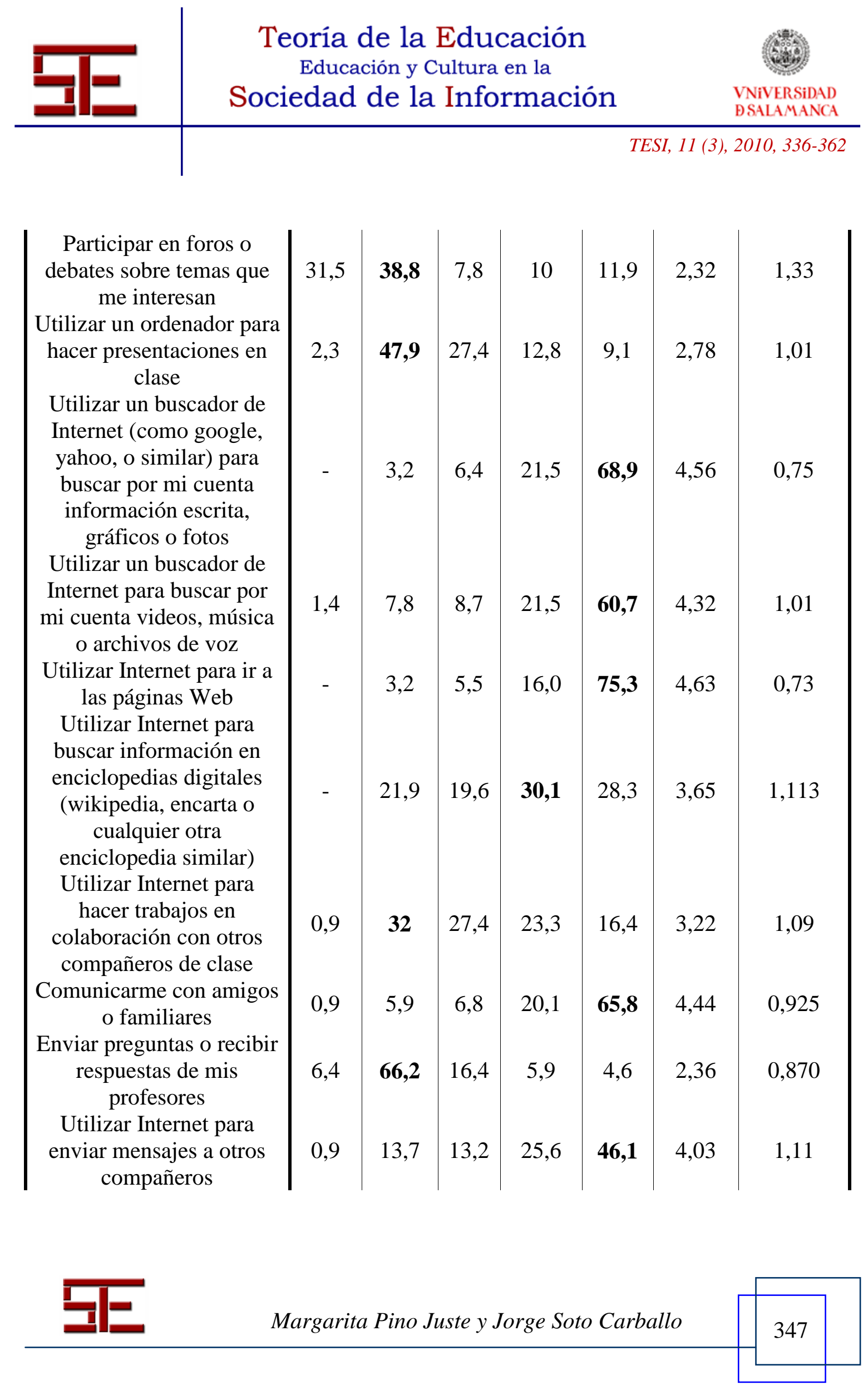


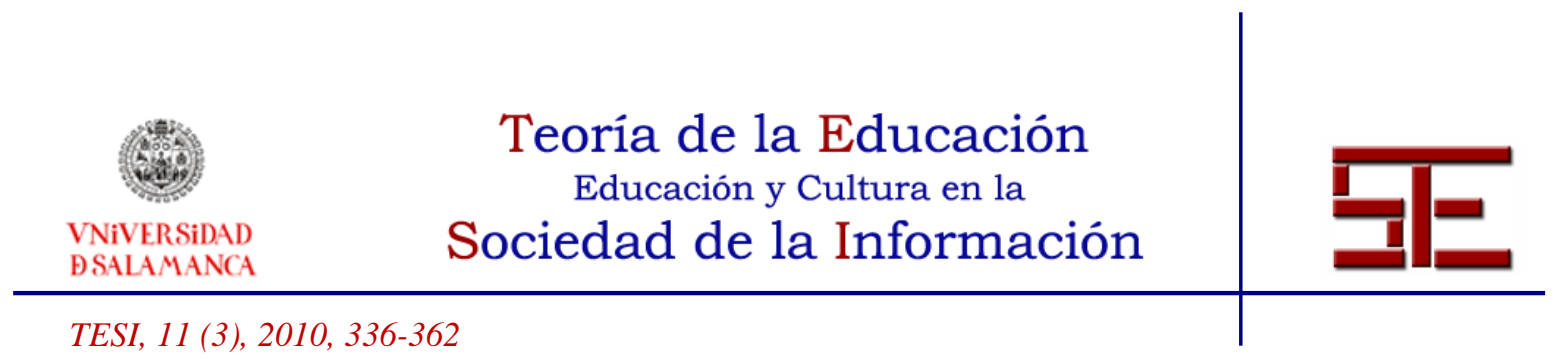

\begin{tabular}{|} 
Utilizar Internet para \\
publicar en una pagina \\
Web, Blog o revista \\
electrónica \\
Utilizar Internet para \\
compartir con mis \\
compañeros materiales \\
relacionados con alguna \\
asignatura \\
Participar en foros o \\
debates sobre temas que \\
me interesan \\
Leer periódicos o revistas \\
digitales \\
Escuchar programas de \\
radio o podcats \\
Leer mis Blogs favoritos \\
Compartir fotos, videos u \\
otro tipo de archivos que \\
yo mismo he creado \\
(Youtube, Flickr,...)
\end{tabular}

\begin{tabular}{|l|c|c|c|c|c|c|}
$\mathbf{3 3 , 3}$ & 21,9 & 10,5 & 14,2 & 19,6 & 2,65 & 1,54 \\
14,2 & 23,7 & 12,3 & 18,7 & $\mathbf{3 1 , 1}$ & 3,29 & 1,47 \\
& & & & & & \\
32,9 & $\mathbf{4 0 , 2}$ & 7,8 & 9,6 & 8,7 & 2,20 & 1,24 \\
$\mathbf{1 9 , 6}$ & $\mathbf{3 7}$ & 10,5 & 12,3 & 20,1 & 2,76 & 1,43 \\
$\mathbf{3 6 , 1}$ & 32 & 11,9 & 11,9 & 7,8 & 2,23 & 1,27 \\
23,3 & $\mathbf{3 1 , 1}$ & 13,7 & 13,7 & 18,3 & 2,73 & 1,43 \\
$\mathbf{2 3 , 7}$ & 24,2 & 12,3 & 18,3 & 21,5 & 2,89 & 1,49
\end{tabular}

Tabla 5: Frecuencia de utilización de las diferentes herramientas

Leyenda: $1=$ Nunca; $2=$ Casi Nunca; $3=$ Una vez a la semana; 4=2 o 3 veces a la semana; 5=Diariamente

Como vemos el alumnado utiliza habitualmente el ordenador para ir a páginas Web, buscar información en la red, comunicarse con los amigos y familiares y enviar mensajes. Utiliza Internet y el ordenador cada semana para bajar películas, juegos o programas, para buscar información en enciclopedias digitales, para escribir y realizar gráficos o tablas, para trabajar en colaboración con los compañeros y compartir con sus compañeros materiales relacionados con alguna asignatura. Y utilizan esporádicamente el ordenador para jugar en línea, compartir fotos, entrar en foros, hacer preguntas al profesor o publicar sus propias páginas.

Analizados estos resultados en los grupos de investigación las conclusiones han resultado evidentes. Normalmente el alumnado manifiesta utilizar el ordenador e Internet diariamente como herramienta de ocio y en segundo lugar, y por lo tanto

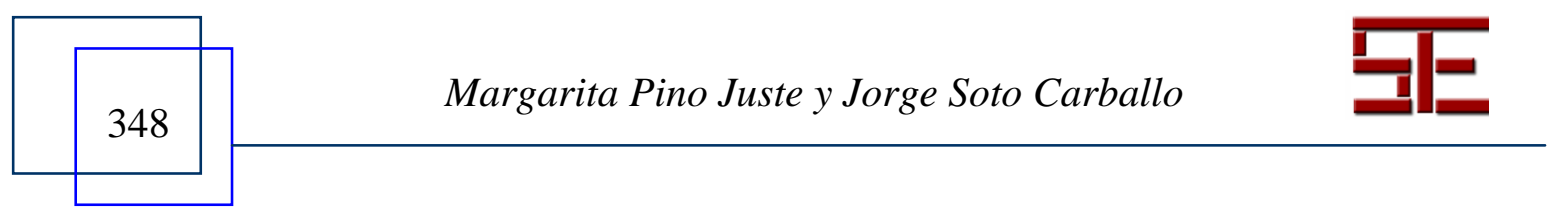




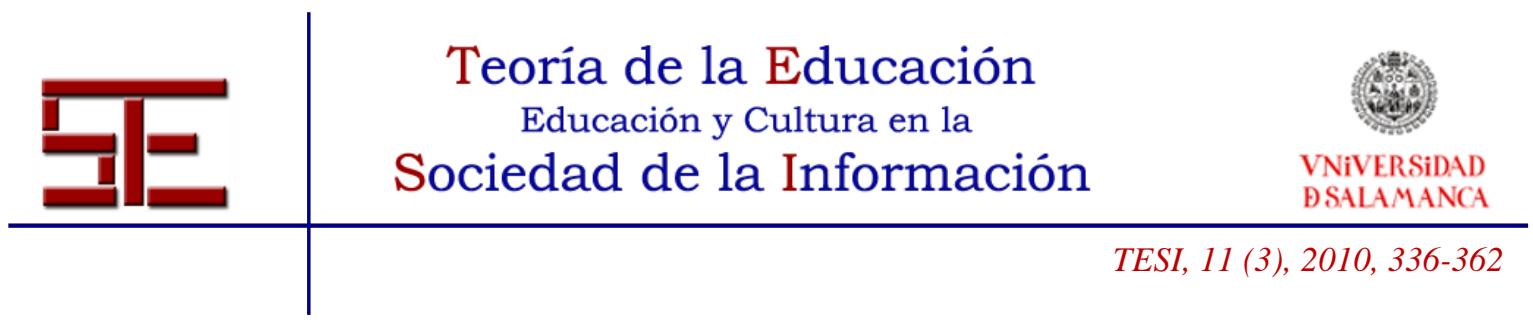

cuando lo necesita, como herramienta de trabajo intelectual. Cuando el ordenador se utiliza para el primer fin el alumnado lo hace a cualquier hora y no existe pereza para conectarse. Para el segundo fin normalmente se hace se forma obligada $y$ cuando no hay más remedio.

Aunque en los grupos de discusión no hay ningún alumno que no asista regularmente a clase, el profesorado opina que el alumnado que no asiste a clase suele utilizar más el correo para comunicarse con el profesor y suele entregar sus actividades antes que el grupo que asiste a clase.

Con respecto a las actitudes y motivaciones vemos que las medias están siempre cerca de 4 y 5 lo que significa que están bastante o muy de acuerdo con cada aseveración salvo en los ítems presentados de forma negativa.

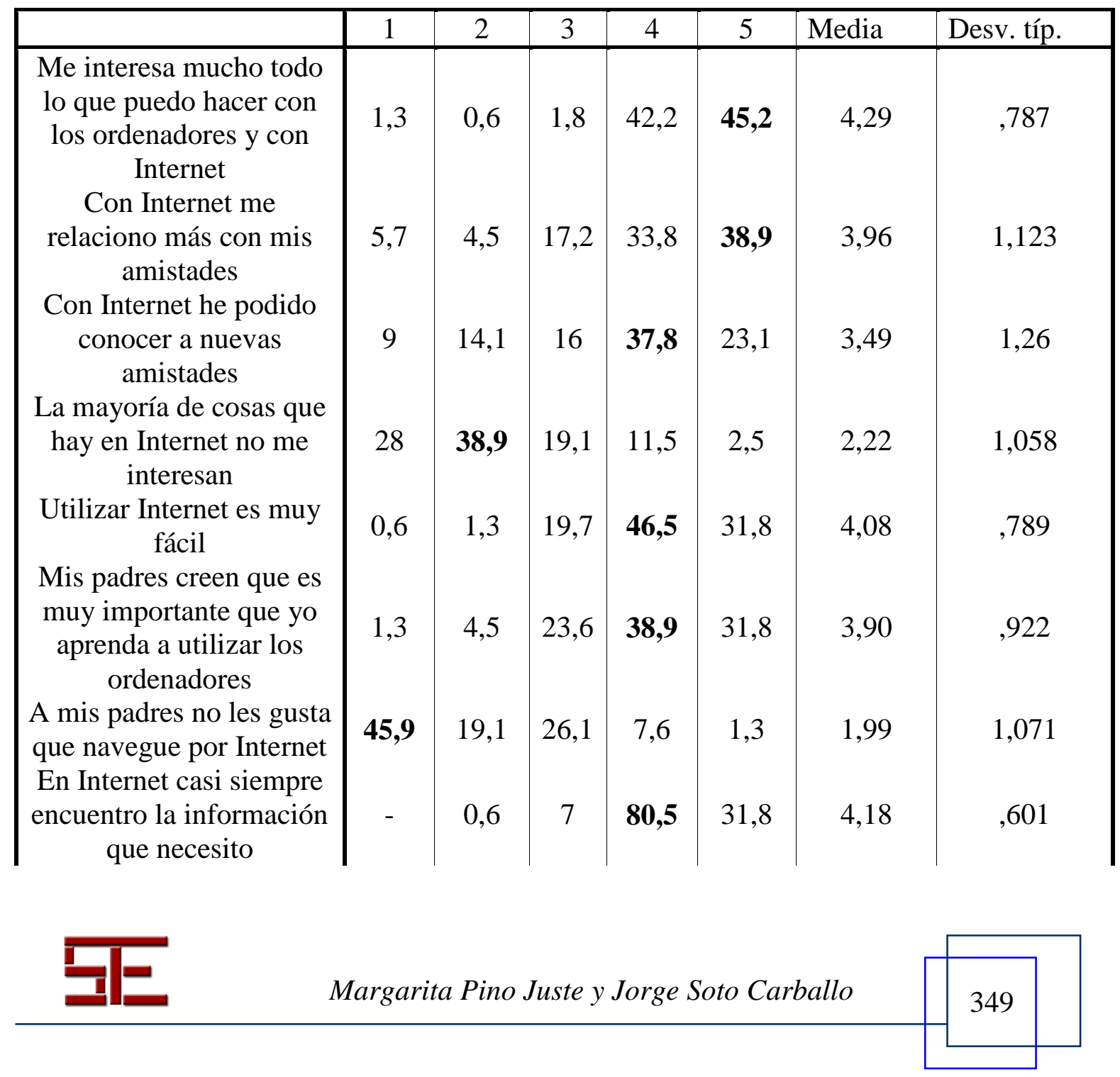




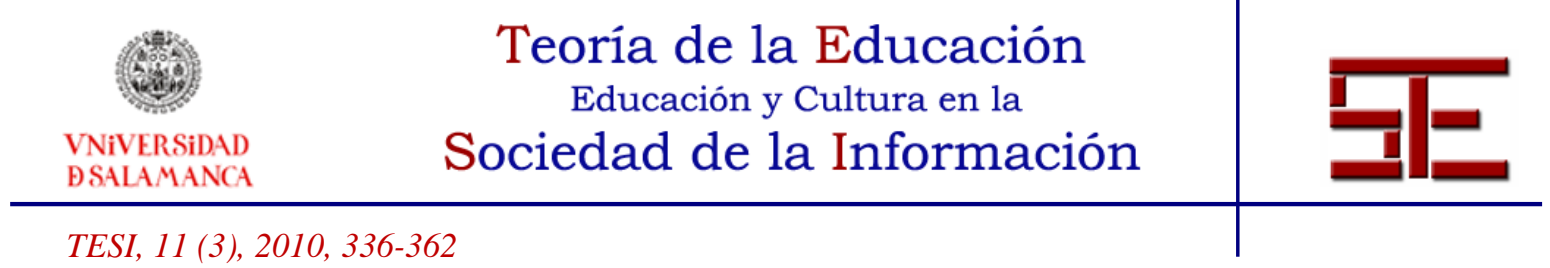

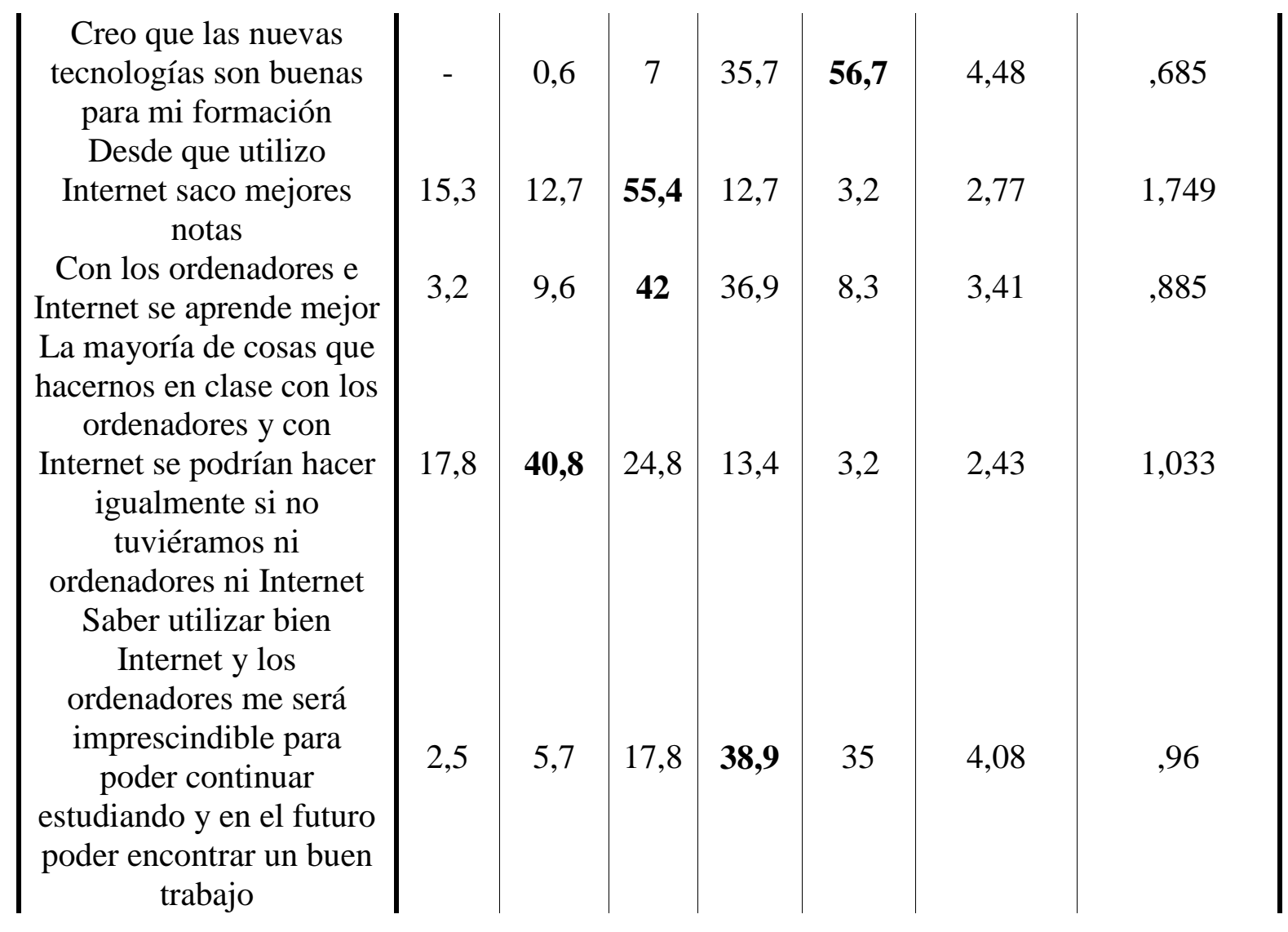

Tabla 6: Motivaciones y creencias sobre las TIC

Leyenda: 1=Muy en desacuerdo; 2=Bastante en Desacuerdo; $3=$ Ni de acuerdo, ni en desacuerdo; 4=Bastante de acuerdo; 5=Muy de acuerdo

Se destaca el interés sobre lo que pueden hacer con los ordenadores, consideran que las nuevas tecnologías son buenas para su formación, que con Internet se relacionan con los demás y que Internet es imprescindible para sus estudios y para conseguir un mejor futuro laboral. Y están en desacuerdo con la idea de que a sus padres no les gusta que naveguen en Internet.

Por lo que respecta al dominio, tanto cognoscitivo como técnico, de las diferentes destrezas relacionadas con el dominio de las TIC nos encontramos con los siguientes datos:

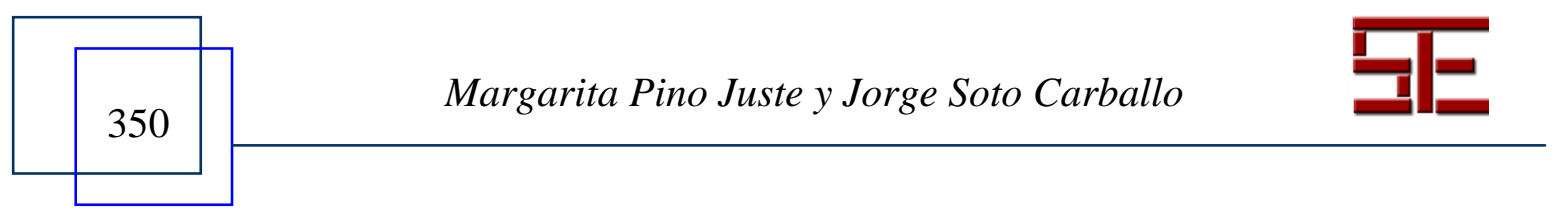




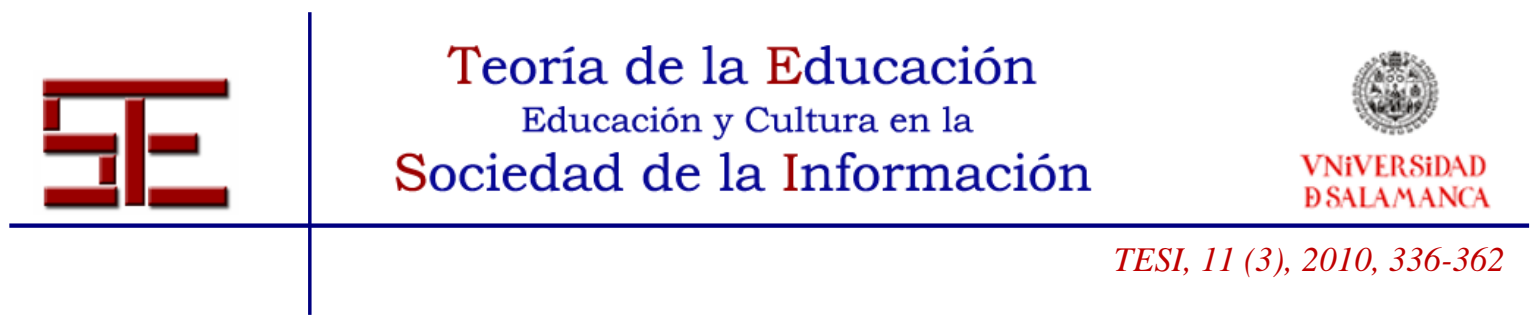

\begin{tabular}{|c|c|c|c|c|c|c|c|c|}
\hline & Dimensión & 1 & 2 & 3 & 4 & 5 & Media & Desv. típ. \\
\hline $\begin{array}{c}\text { Realización de } \\
\text { actividades académicas } \\
\text { y formativas con las TIC }\end{array}$ & TI & 3,9 & 26,8 & 31,4 & 27,5 & 10,5 & 3,06 & 1,044 \\
\hline Bajar archivos & A & 2,6 & 14,1 & 23,7 & 35,3 & 24,4 & 3,65 & 1,076 \\
\hline Crear un documento & A & 7,1 & 28,8 & 32,1 & 25 & 7,1 & 2,96 & 1,053 \\
\hline Usar buscadores & $\mathrm{A}$ & 3,2 & 7,1 & 18,6 & 23,1 & 48,1 & 4,06 & 1,114 \\
\hline $\begin{array}{c}\text { Analizar críticamente } \\
\text { páginas Web }\end{array}$ & $\mathrm{TD}$ & 3,1 & 44,5 & 11,6 & 7,1 & 5,8 & 2,12 & 1,107 \\
\hline $\begin{array}{l}\text { Realizar actividades } \\
\text { lúdicas y de ocio }\end{array}$ & A & 6,5 & 12,9 & 13,5 & 21,9 & 45,2 & 3,86 & 1,295 \\
\hline $\begin{array}{c}\text { Realizar actividades } \\
\text { laborales }\end{array}$ & A & 28,2 & 31,4 & 17 & 16,3 & 6,5 & 2,41 & 1,243 \\
\hline $\begin{array}{l}\text { Realizar actividades de } \\
\text { gestión y administración }\end{array}$ & TI & 39,1 & 37,8 & 9,6 & 5,8 & 7,7 & 2,05 & 1,190 \\
\hline $\begin{array}{l}\text { Utilizar el ordenador } \\
\text { como herramienta de } \\
\text { comunicación }\end{array}$ & TD & 18,6 & 23,7 & 20,5 & 17,9 & 19,2 & 2,96 & 1,393 \\
\hline $\begin{array}{l}\text { Realizar actividades de } \\
\text { autoformación }\end{array}$ & TI & 16 & 34,6 & 25,6 & 15,4 & 8,3 & 2,65 & 1,168 \\
\hline $\begin{array}{l}\text { Aprender a trabajar en } \\
\text { equipo en entornos } \\
\text { colaborativos }\end{array}$ & TI & 18,7 & 43,2 & 25,8 & 7,7 & 4,5 & 2,36 & 1,019 \\
\hline $\begin{array}{c}\text { Actualizar } \\
\text { conocimientos sobre los } \\
\text { medios }\end{array}$ & TD & 5,1 & 20,5 & 32,7 & 23,7 & 17,9 & 3,29 & 1,136 \\
\hline $\begin{array}{l}\text { Aprovechar nuevos } \\
\text { entornos virtuales de } \\
\text { aprendizaje }\end{array}$ & A & 2,6 & 14,7 & 35,9 & 31,4 & 15,4 & 3,42 & 1,003 \\
\hline $\begin{array}{l}\text { Evaluar críticamente } \\
\text { recursos educativos }\end{array}$ & TD & 111,7 & 24,7 & 33,8 & 21,4 & 8,4 & 2,90 & 1,125 \\
\hline $\begin{array}{c}\text { Trabajar de manera } \\
\text { individual }\end{array}$ & TI & 1,3 & 9,6 & 25 & 23,7 & 40,4 & 3,92 & 1,075 \\
\hline $\begin{array}{l}\text { Evaluar críticamente la } \\
\text { información recogida }\end{array}$ & TD & 5,8 & 16 & 25,6 & 28,8 & 23,7 & 3,49 & 1,183 \\
\hline
\end{tabular}

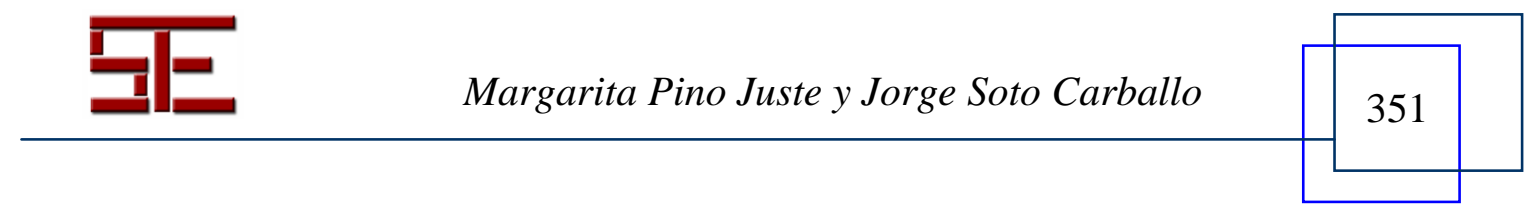



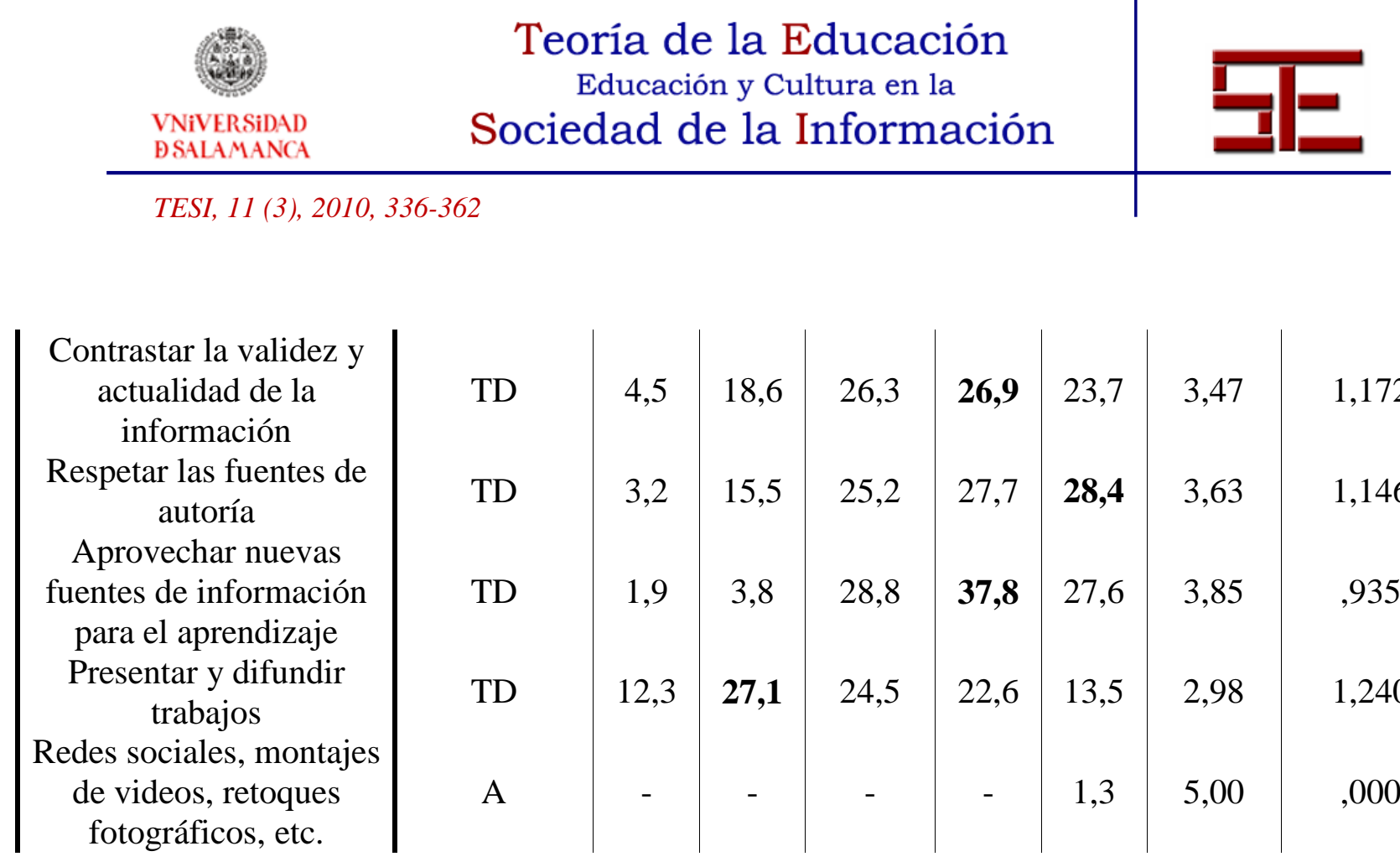

\begin{tabular}{|c|c|c|c|c|c|c|c|}
\hline TD & 4,5 & 18,6 & 26,3 & 26,9 & 23,7 & 3,47 & 1,172 \\
\hline TD & 3,2 & 15,5 & 25,2 & 27,7 & 28,4 & 3,63 & 1,146 \\
\hline TD & 1,9 & 3,8 & 28,8 & 37,8 & 27,6 & 3,85 & ,935 \\
\hline TD & 12,3 & 27,1 & 24,5 & 22,6 & 13,5 & 2,98 & 1,240 \\
\hline A & - & - & - & - & 1,3 & 5,00 & ,000 \\
\hline
\end{tabular}

Tabla 7: Dominio de las TIC

Leyenda: $\mathrm{TI}=$ competencias de los medios (TIC) como Técnicas de trabajo intelectual; $\mathrm{A}=$ Alfabetización tecnológica; $\mathrm{TD}=$ Competencias en el Tratamiento y difusión de la información

1=Nunca; 2=Casi Nunca; 3= Una vez a la semana; 4=2 o 3 veces a la semana; $5=$ Diariamente

Como vemos, los ítems hacen referencia a tres dimensiones: alfabetización tecnológica, instrumentos de trabajo intelectual y tratamiento y difusión de la información y herramientas de comunicación.

Se comprueba que en los ítems que se refieren a la alfabetización tecnológica el alumnado realiza actividades como bajar archivos, crear documentos, utilizar buscadores y sobre todo realizar actividades lúdicas y de ocio mayoritariamente 2 o 3 veces a la semana e incluso diariamente, salvo en lo que se refiere a actividades laborales (todavía están estudiando y pocos trabajan) y al aprovechamiento de entornos virtuales.

También son bajos los porcentajes referidos a las competencias de los medios como técnicas de trabajo intelectual. Los alumnos realizan actividades académicas y trabajos individuales alguna vez a la semana, pero casi nunca realizan actividades de gestión y administración (matricula virtual, gestión de becas, consulta de notas de las materias, etc.), ni trabajos colaborativos, ni actividades de autoformación o autoaprendizaje.

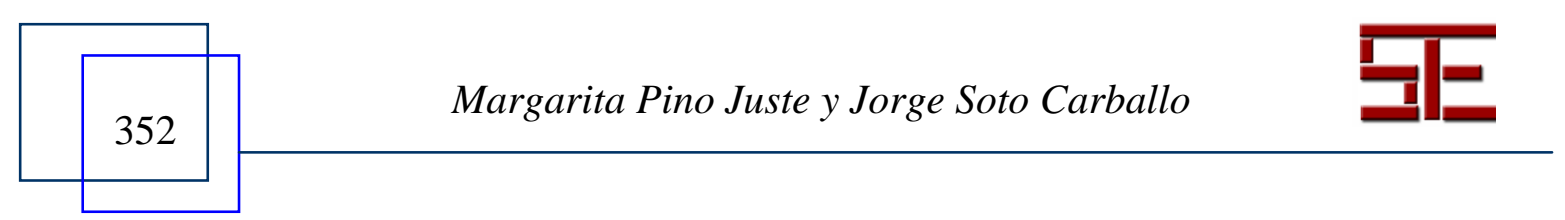




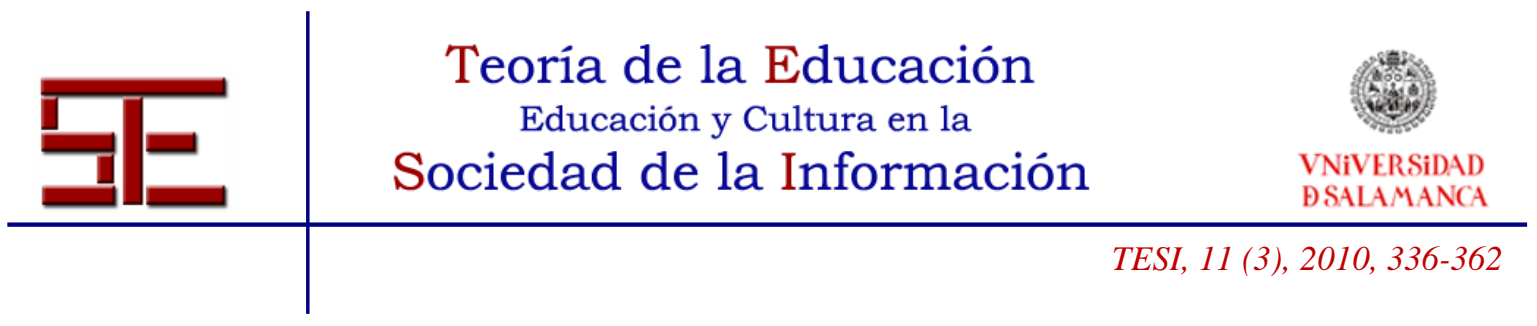

Por lo que respecta a las competencias referidas al tratamiento y difusión de la información y herramientas de comunicación nos encontramos con mucha variabilidad en los datos. El alumnado reconoce que casi nunca realiza una evaluación crítica sobre los recursos de Internet, pero si admite realizar esa evaluación sobre la información recogida contrastando la validez y actualidad de la misma admitiendo aprovechar las fuentes de información y recursos que le facilitan las TIC para su propio aprendizaje. Cuando les preguntamos si consideran imprescindible el dominio de herramientas digitales para su formación contestan afirmativamente el 96\%. No obstante, cuando preguntamos si se sienten preparados para utilizar estas herramientas en el proceso de enseñanza-aprendizaje con sus futuros alumnos el $82 \%$ se manifiesta negativamente. De los que contestan afirmativamente solo el $15 \%$ dice que está muy preparado y que podría hacerlo. La media es de 3,22.

En los grupos de discusión se resalta que quizás influya en el dominio de estas competencias las actitudes de los profesores y las preferencias que transmiten durante el proceso de enseñanza-aprendizaje en el aula. En este sentido el profesorado muestra poca seguridad técnica y didáctica encontrándose en su quehacer profesional con auténticos problemas para poder desarrollar competencias en el uso de los medios de información y comunicación de manera que puedan proporcionar a los alumnos ayuda y formación a pesar de que en general los profesores afirman que tiene confianza en las nuevas tecnologías para desarrollar estilos docentes más personalizados y flexibles a la vez que mejorar la participación. Según el alumnado la mayoría de profesores no manejan con suficiente habilidad los medios, hacen comentarios negativos durante la clase sobre la utilización de los mismos y parecen estar obligados a utilizarlos. La mayoría de ellos utiliza el ordenador para la presentación en PowerPoint del contenido de su materia como apoyo en la clase magistral y en la plataforma digital se suben los documentos para su lectura.

Exponen los alumnos: "Sabemos más nosotros que ellos del manejo del ordenador y se pasan el día despotricando sobre la pérdida de tiempo que generan los medios" (AEP12).

"Las únicas novedades son que ahora en vez de ir a la fotocopiadora te bajas los textos de la plataforma, pero no aprendes a utilizar esos medios para cuando tu seas profesor y tengas que utilizarlos"(AEF3).

El alumnado considera que no hay una formación específica sobre la explotación didáctica de los medios.

Para los docentes la falta de tiempo, la carencia de personal especializado que apoye cuando se producen problemas, la falta de recursos y la sensación de que la

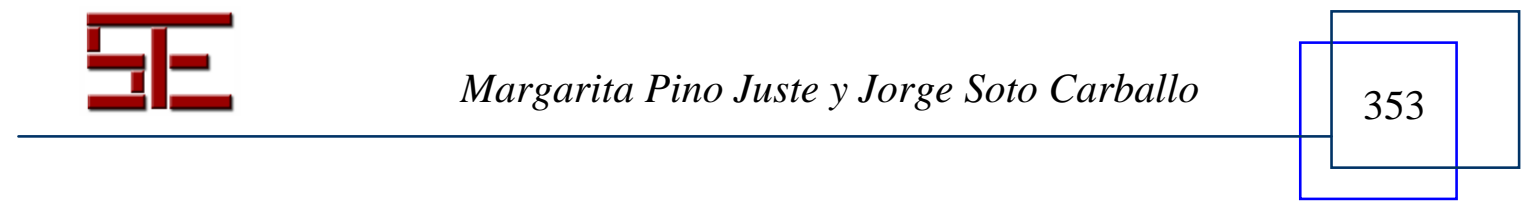




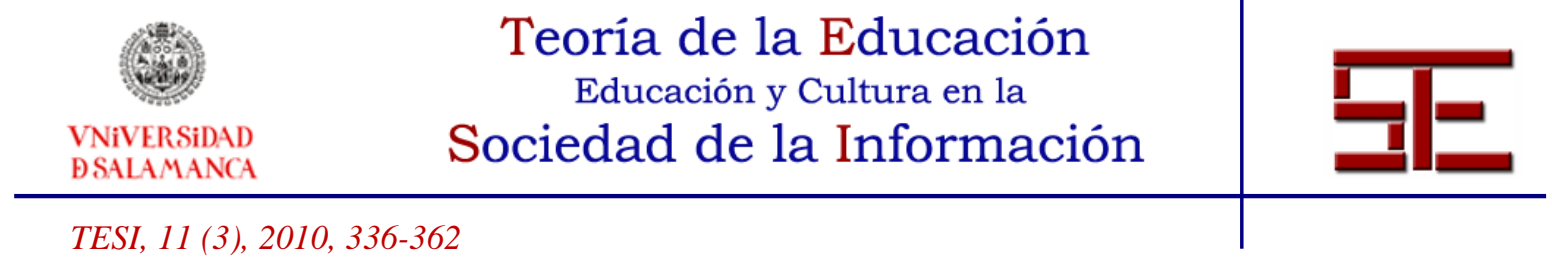

utilización de estos recursos no mejora el aprendizaje, son las principales dificultades que encuentran a la hora de implicarse en la implementación de estas herramientas. La formación que demandan los profesores supone uno de los principales escollos con los que se encuentran en su vida laboral habitual. Señala un profesor: "Nadie te enseña a utilizar los medios, todo depende de ti. Ahora esta de moda una nueva herramienta y tenemos que utilizarla sin formación alguna y ni siquiera sabemos si es más eficaz o no que lo que hacíamos hasta ahora" (PEP1). Los principales problemas que el alumnado se encuentra para la utilización de las TIC en el aula los podemos comprobar en la siguiente Tabla.

\begin{tabular}{|c|c|c|c|c|c|c|c|}
\hline & 1 & 2 & 3 & 4 & 5 & Media & Desv. típ. \\
\hline Software & 3,8 & 10,9 & $\mathbf{3 8 , 5}$ & 26,9 & 19,9 & 3,48 & 1,050 \\
Conexión a la red & 7,7 & 24,5 & $\mathbf{2 9 , 7}$ & 27,1 & 11 & 3,09 & 1,125 \\
Hardware & 13,5 & $\mathbf{3 0 , 1}$ & 28,2 & 17,9 & 10,3 & 2,81 & 1,185 \\
Problemas en la red & 21,2 & $\mathbf{3 2 , 1}$ & 21,2 & 17,3 & 8,3 & 2,60 & 1,233 \\
eléctrica & & & & & & & \\
Intromisión de aspectos no & 23,7 & $\mathbf{2 6 , 3}$ & 18,6 & 15,4 & 16 & 2,74 & 1,396 \\
deseados & $\mathbf{2 7 , 6}$ & 23,1 & 21,8 & 12,8 & 14,7 & 2,64 & 1,391 \\
Carencia de recursos & $\mathbf{2 9 , 5}$ & 23,7 & 26,3 & 12,8 & 7,7 & 2,46 & 1,251 \\
Experiencia en las TIC & 11 & 17,4 & 18,7 & $\mathbf{3 0 , 0}$ & 22,6 & 3,36 & 1,304 \\
limitada & 8,4 & 9,7 & $\mathbf{2 9 , 7}$ & 27,1 & 25,2 & 3,51 & 1,208 \\
Cansancio visual & 1,6 & - & 7,6 & 29,2 & $\mathbf{3 4 , 2}$ & 3,65 & 1,132 \\
Logística del aula & & & &
\end{tabular}

Tabla 8: Obstáculos para la utilización de las TIC en el aula

Como se comprueba, destacan los excesivos problemas con el software sobre todo: baja velocidad, incompatibilidades, funcionamientos anómalos, complejidad en el uso de los programas, "cuelgues" del sistema, falta de formación del profesorado, etc. Destaca también el cansancio visual y la logística del aula ya que hay restricción de horarios de acceso, pocos ordenadores en función del número de alumnos, etc. Podemos puntualizar, sin embargo, que estos problemas no son muy habituales ya que ninguno de los ítems llega a una media de 4.

En los grupos de discusión se evidencian las dificultades para el tratamiento de la información que se recoge utilizando las TIC. El profesorado apunta las dificultades de

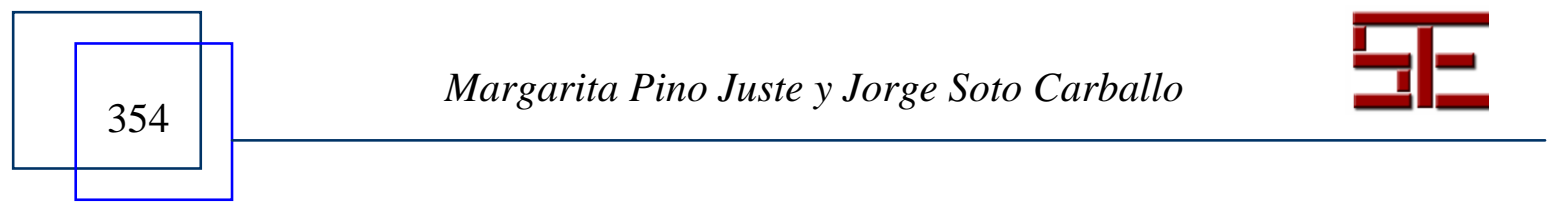




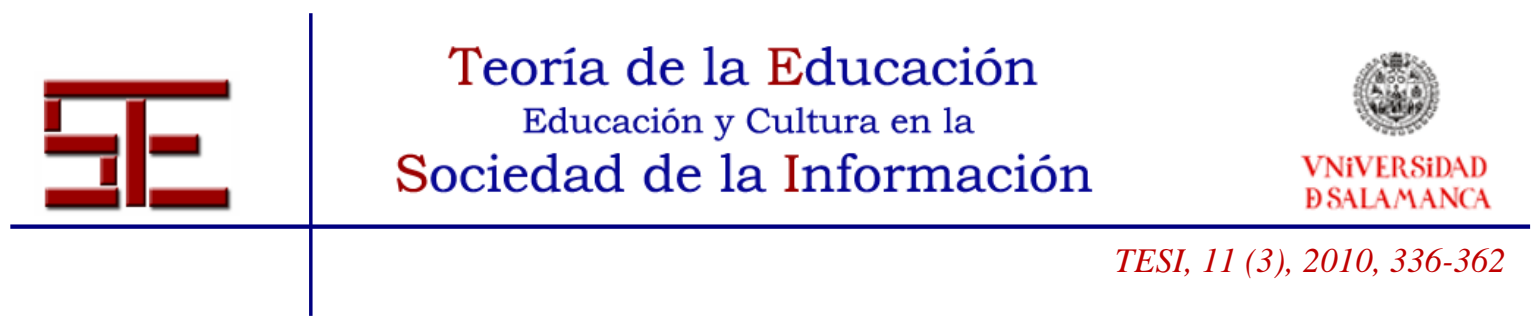

argumentación, análisis y síntesis que tiene el alumnado para organizar la información, así como la escasez de análisis crítico y reflexivo que se realiza.

El alumnado por su parte manifiesta la gran cantidad de información que deben analizar y que pocas veces se les ofrecen pautas para que guíen el trabajo de análisis. Desconocen lo que es importante y cuáles son los objetivos que deben perseguir.

Del análisis de las submuestras en función del género, edad, especialidad y de cuando se ha adquirido la formación en las TIC se infieren opiniones similares, es decir, estadísticamente no existe diferencia significativa entre las medias de los diferentes colectivos. Ello puede deberse a la homogeneidad de la muestra.

\section{4.- CONCLUSIONES Y DISCUSIÓN}

Son evidentes las limitaciones del estudio relacionadas con la generalización de los resultados ya que, dada la metodología utilizada y el número de sujetos de la muestra, no es posible realizar ningún tipo de inferencia estadística. Sin embargo, el estudio permite la generalización analítica, es decir la ampliación de los planteamientos teóricos existentes en función de la evidencia obtenida. Sería muy importante replicar este estudio en contextos similares utilizando otro tipo de metodologías que permitan la generalización estadística.

A pesar de ello, del estudio realizado podemos concluir que, en general, el alumnado no tiene una formación específica sobre el funcionamiento de los ordenadores.

Sus actitudes hacia las TIC son muy positivas y sus motivaciones se centran fundamentalmente en que las tecnologías son útiles para mejorar su aprendizaje y para su futuro laboral. Recordemos que las actitudes que muestra el profesorado hacia las TICs es uno de los principales predictores de aceptación o rechazo de su uso en el proceso de enseñanza (Bullock, 2004). Estas actitudes positivas están justificadas por las potencialidades que les atribuyen de motivación, interés y refuerzo de los aprendizajes y por la convicción de que no serán reemplazados por los ordenadores, ni disminuirá su protagonismo dentro del aula (Cuadrado y Fernández, 2000; Albirini, 2006). Según García-Valcárcel (2003), si los docentes perciben que el uso de este tipo de tecnologías les exigirá importantes cambios en su metodología y modificación de gran parte de sus rutinas, aumentará la probabilidad de aparición de sentimientos de ansiedad y estrés y, consecuentemente, la emergencia de actitudes negativas hacia su uso en el aula. Esta ansiedad y estrés no vienen provocados tanto por el exceso de trabajo, cuanto por la inseguridad e inestabilidad que supone tener que romper con las rutinas que durante años les han ayudado a dominar la situación (Tejedor y GarcíaValcárcel, 2006).

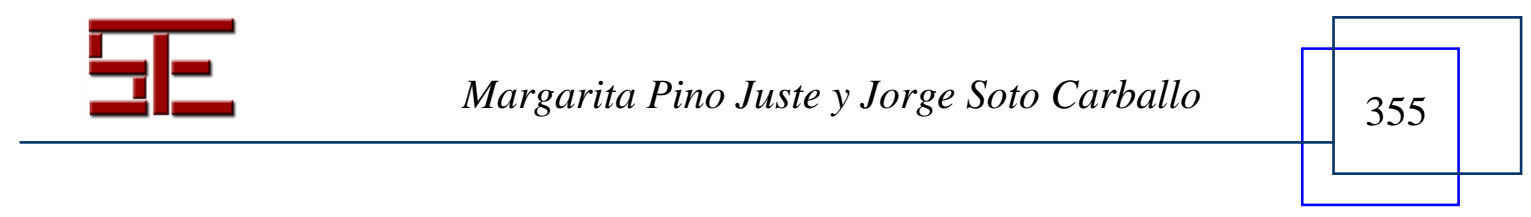




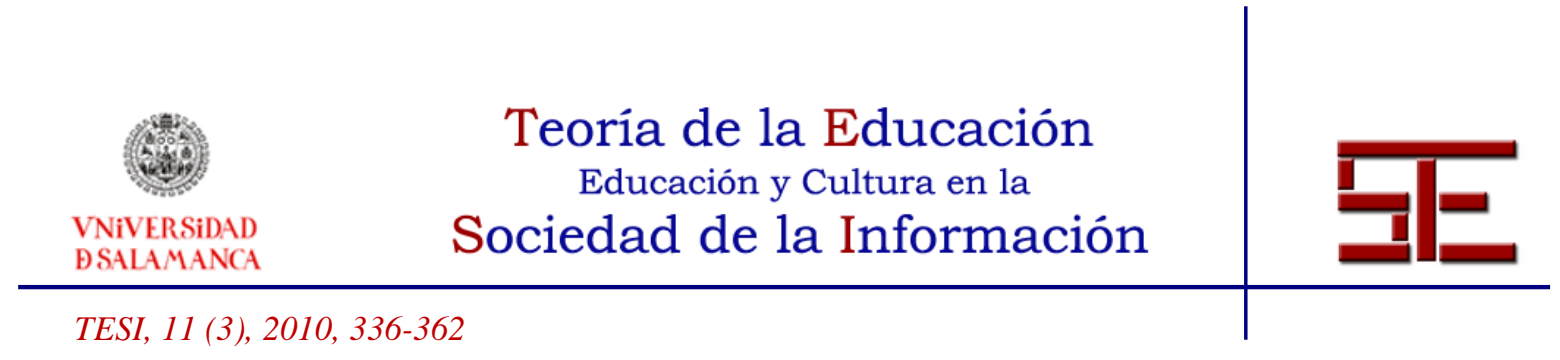

Por la contra, las actitudes negativas conllevaría limitaciones en su implementación (Karsenti, 2004).

El alumnado realiza actividades sin ayuda como bajar archivos, crear o imprimir documentos, enviar un e-mail, utilizar buscadores y sobre todo realiza actividades lúdicas y de ocio aunque escasean actividades de gestión y administración, trabajos colaborativos o actividades de autoformación siendo muy limitada la realización de una evaluación crítica sobre los recursos de Internet. Necesita ayuda para las actividades menos habituales como crear una base de datos, una hoja de cálculo, diseñar páginas Web o utilizar paquetes estadísticos. Asimismo aprovecha las fuentes de información y recursos que le facilitan las TIC para su propio aprendizaje aunque raramente utilizan los ordenadores en el aula. Solamente en algunas materias y siempre a través de la plataforma digital que pone a su disposición la universidad.

El correo electrónico es utilizado como una herramienta de trabajo siendo el medio por el cual se comunican con los profesores; mientras que la mensajería y las redes sociales las utilizan más el fin de semana y en su tiempo libre como herramienta de comunicación con los amigos.

Debemos tener en cuenta que según Windchitl y Sahl (2002), la utilización de las TIC en el proceso de enseñanza-aprendizaje está directamente relacionada con el conocimiento que poseen a nivel de usuario, las actitudes que presentan ante las TICs y ante el desarrollo tecnológico en general y, la percepción que tengan de la utilidad y potencial pedagógico de las TICs. De ahí que sea importante que el alumno considere las TIC como una herramienta que tiene que dominar para su futuro laboral.

Entendemos, por lo tanto, que dominan destrezas básicas sin embargo no saben sacar rendimiento didáctico de las mismas. En esta línea debemos tener en cuenta que algunos resultados de investigaciones sugieren que la formación en nuevas tecnologías de la información y comunicación ha tenido cierto éxito para aumentar el grado de confianza de los profesores en el uso de los ordenadores en la enseñanza, sin embargo este éxito ha sido atemperado por un grado considerable de reacción negativa hacia la forma y el contenido de la formación (Galanouli, Murphy \& Gardner, 2004).

Quizás influya en el dominio de estas competencias las actitudes de los profesores y las preferencias que transmiten durante el proceso de enseñanza-aprendizaje en el aula. De hecho, los resultados obtenidos en el estudio de Gargallo López, Suárez Rodríguez y Almerich Cerveró (2006) indican que una actitud positiva se asocia con una mayor utilización de las nuevas tecnologías, un uso de superior calidad y una mayor implicación de los alumnos más avanzados. Una actitud negativa se relaciona con menor manejo de estos medios, aunque los datos no son del todo concluyentes.

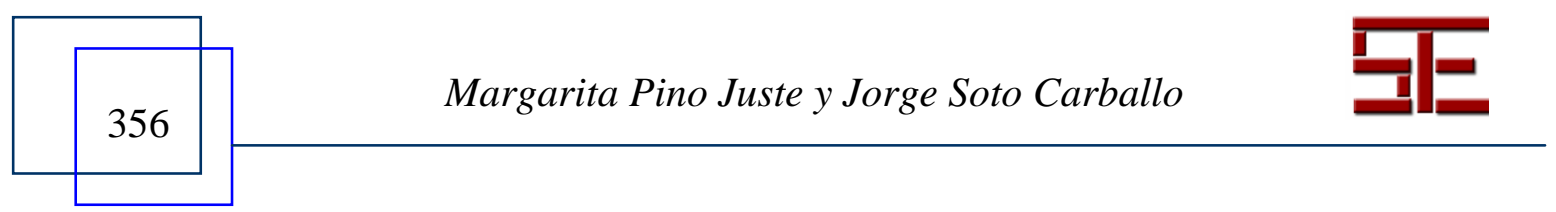




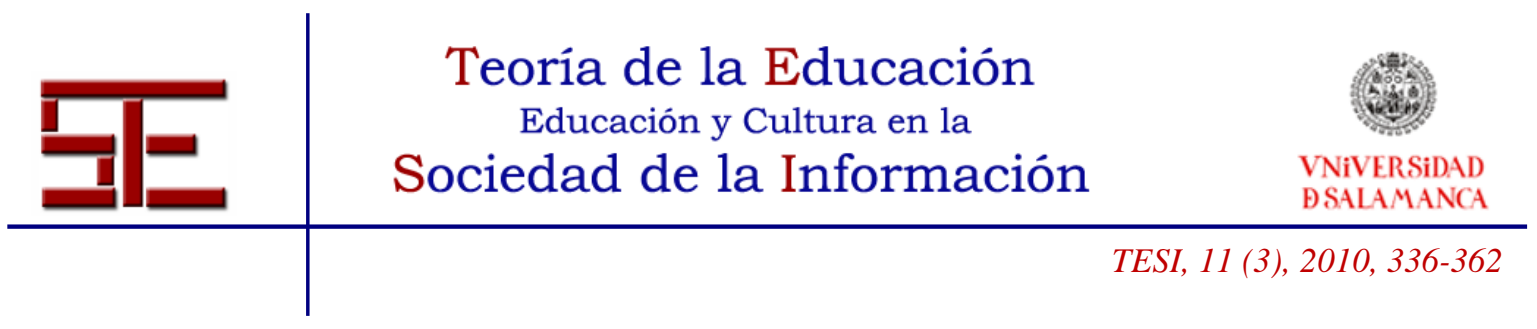

En el estudio de Barton y Haydn (2006) o Haydn y Barton (2007) se destaca que los estudiantes de magisterio no consideraban provechosa la inversión en tiempo y recursos en relación con su formación. En particular señalan que solamente han sido abrumados con mucha información sobre las TIC. Sin embargo, los estudiantes admiten el eficaz impacto que ha tenido el manejo habitual de las TIC durante su formación en el futuro dominio de las mismas.

En nuestro estudio la mayoría del alumnado manifiesta no estar preparado para utilizar las herramientas digitales en el proceso de enseñanza-aprendizaje y sin embargo lo consideran muy importante para su formación.

Estos resultados son similares a estudios realizados con docentes en activo quienes se muestran convencidos de las ventajas y beneficios que sobre el aprendizaje del alumno obtendrían al utilizar las TIC; sin embargo su escasa experiencia y conocimiento sobre sus aplicaciones educativas les genera inseguridad y en muchos casos sentimientos de tecnofobia (Rosen y Weil, 1995; Finlayson y Perry, 1995; Francis-Pelton y Pelton, 1996). De hecho, algunos estudios (Watson, 1997; Strudler et al., 1999, Albirini, 2006) señalan que los docentes no manifiestan competencias para trabajar con las TICs en el aula.

En referencia a los obstáculos los más habituales tiene relación con el software, la logística del aula y el cansancio visual. A similares conclusiones se llega en el estudio de Hammond y otros (2009) cuando indican que el acceso y el dominio de las TIC en el aula son problemas clave que intervienen en la correcta utilización de estas herramientas. Igualmente importante, parece ser la creencia en que las TIC facilitarían una mejora en el proceso de enseñanza-aprendizaje y una mayor voluntad para "aprender a hacer". Consideramos que estas problemáticas se pueden resolver mejorando las infraestructuras del centro.

Otro aspecto destacado es el desconocimiento del profesorado del manejo de las TIC. Ya en otros estudios (Mutton, Mills y McNicholl, 2006) se manifiesta que los tutores sienten que tienen menos dominio de las TIC que sus estudiantes y por lo tanto se manifiestan menos dispuestos a trabajar esta competencia genérica en sus materias ya que no son capaces de ofrecer el apoyo necesario a su alumnado. Los autores sugieren que se deberían ofrecer ventajas a los tutores que favorezcan la utilización de las TIC en el aula para facilitar los procesos de innovación.

En este sentido, numerosos informes nos advierten de que los docentes no se sienten capacitados para incorporar las TICs al proceso de enseñanza-aprendizaje de manera eficaz (OECD, 2001; ISTE, 2002; Comisión Europea, 2002; BECTA, 2004).

Para evitar los obstáculos detectados sería importante tal como señala Ottesen (2006) que existiera una mayor interacción en las relaciones personales e institucionales ya que

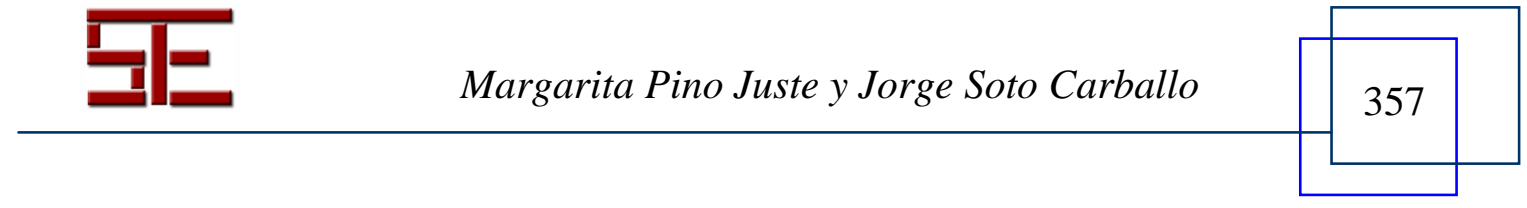




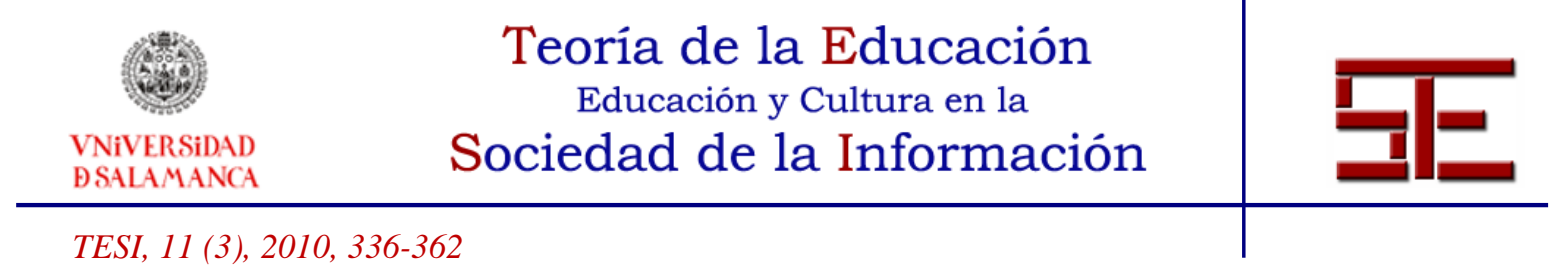

esta mejoraría el horizonte para el desarrollo de los programas de enseñanza y aprendizaje con las TIC.

El trabajo con las TIC requiere, a nuestro juicio, una mayor capacidad creativa para la resolución de los problemas complejos, la adquisición de una cultura de argumentación, poner en práctica nuevas ideas y formas de organización, enfatizar las soluciones y resultados en colaboración y una evaluación de calidad. Y, sobre todo exige cambios hacia una cultura que aprende (tutores acompañantes, mayor calidad didáctica, proyectos conjuntos de universidad y escuela).

De ahí que los retos que debemos afrontar en la Sociedad de la Información estén condicionados por políticas educativas que favorezcan una formación del profesorado en competencias digitales que conlleve un dominio de estas tanto técnico como didáctico y por el apoyo a la investigación para la creación de nuevas plataformas que respondan a necesidades pedagógicas concretas, así como al tratamiento en el empleo de tecnología como un activador importante para abrir nuevos caminos en la construcción de habilidades y desarrollo personal.

\section{5.- BIBLIOGRAFÍA.}

Adell, J. (1998). Nuevas Tecnologías e innovación educativa. Organización y Gestión Educativa, 1, 3-7.

Aguado Román, G. (2008). Cómo valorar la calidad de la enseñanza basada en las TIC: pautas e instrumentos de análisis. Barcelona: Editorial Graó.

Albirini, A. (2006). Teachers' attitudes toward information and communication technologies: the case of Syrian EFL teachers. Computers \& Education, 47, 373-398.

Álvarez Castillo, J.L. (2001). Posibilidades y límites político-educativos de las nuevas tecnologías de la información y la comunicación. Revista española de pedagogía, 218, 85-104.

Barquín Ruiz, J. (2005). La implantación de las Tecnologías de la Información en la sociedad y en los centros educativos públicos de la Comunidad de Andalucía. Revista Iberoamericana de Educación, 36, 155-174.

Barton, R. y Haydn, T. (2006). Trainee Teachers' Views on What Helps Them to Use Information and Communication Technology Effectively in Their Subject Teaching. Journal of Computer Assisted Learning, 22 (4), 257-272.

BECTA (British Educational Communications and Technology Agency) (2004). A review of the research literature on barriers to the uptake of ICTby teachers. Extraído el 7 Diciembre, 2009 de http://www.becta.org.uk.

Bullock, D. (2004). Moving from theory to practice: an examination of the factors that preservice teachers encounter as they attempt to gain experience teaching with

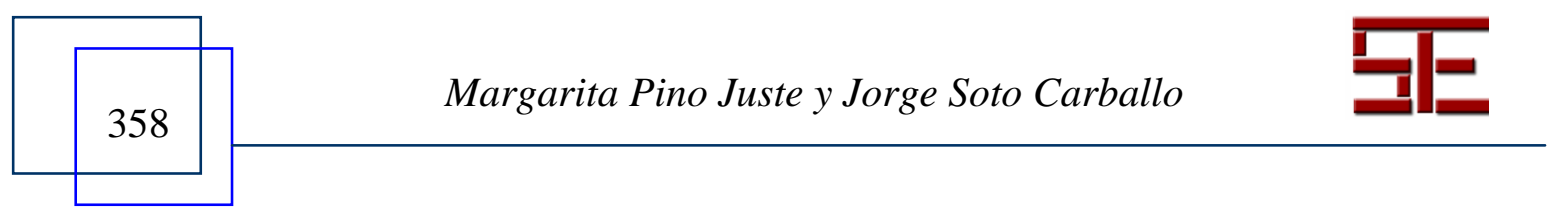




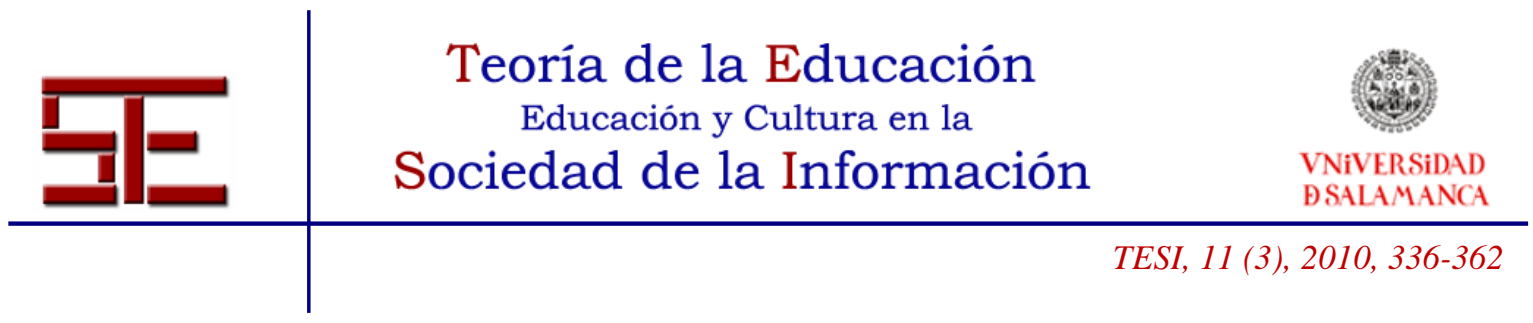

technology during field placement experiences. Journal of Technology and Teacher Education, 12 (2), 211-237.

Cabero, J. y Barroso, J. (Coords) (2007). Posibilidades de la Teleformación en el Espacio Europeo de Educación Superior. Granada: Ediciones Mágina.

Clarke, C. (2004). Opening address to the BETT conference, London, 7 January.

Coll, C.; Mauri, T. e Onrubia, J. (2008). Análisis de los usos reales de las TIC en contextos educativos formales: una aproximación sociocultural. Revista Electrónica de Investigación Educativa, 10 (1). Extraído el 12 Diciembre, 2009 de http://redie.uabc.mx/vol10no1/contenido-coll2.html.

COMISIÓN EUROPEA-EOS GALLUP EUROPE (2002). Les enseignants et la societé de l'information. FLASH EB-119. Bruselas: Comisión Europea. Extraído el 2 agosto, 2009 de http://europa.eu.int/comm/public_opinion/flash/fl119_fr.pdf.

Cuadrado, I. y Fernández, I. (2000). Las actitudes del profesorado ante el ordenador a las puertas del siglo XXI, en MARCHENA E. y ALCALDE C. (Coords.). La perspectiva de la educación en el siglo que empieza. Cádiz: Universidad de Cádiz. pp. 847-850.

Cuadrado, I. y Fernández, I. (2003). Las NTIC en la educación secundaria: ¿Elementos de acercamiento o distanciamiento intergeneracional? En A. Neto et al. Didácticas $e$ Metodologías de Educação. Percursos e Desafios (pp.203-211). Évora: Universidade de Évora.

Cuckle, P., Clarke S. \& Jenkins I. (2000). Trainees' information and communications technology skills and their use during teacher training. Journal of Information Technology for Teacher Education 9, 9-22.

Finlayson, H.M. y Perry, A. (1995). Turning skeptics into missionaries: the case for compulsory information technology courses. Journal of Information Technology for Teacher Education, 4 (3), 351-361.

Fisher, M. (2000) Computer skills of initial teacher education students. Journal of Information Technology for Teacher Education, 9, 109-123.

Francis-Pelton, L. y Pelton, T. (1996). Building attitudes: how a technology course affects pre-service teachers attitudes about technology. Extraído el 9 septiembre, 2009 de http://web.uvic.ca/educ/lfrancis/web/attitudesite.html.

Galanouli, D., Murphy C. \& Gardner, J. (2004). Teachers' perceptions of the effectiveness of ICT-competence training. Computers \& Education, 43 (1-2), 63 - 79.

Gallego Domingo, J. (dir) (2007). Cuestionario educared. Las TIC en el aula 2007. $\begin{array}{lllll}\text { Extraído el } & 22 & \text { octubre, } & 2009 & \text { de }\end{array}$ http://www.educared.net/images/ficheros/weblethtml/5523/encuesta2007.pdf .

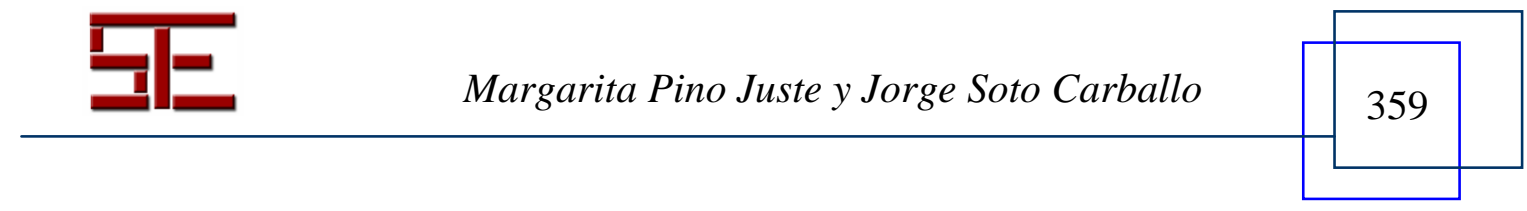




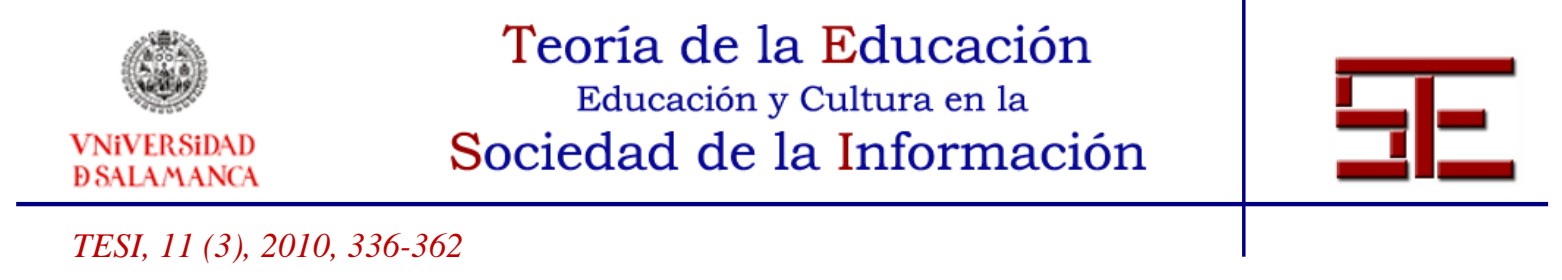

García Aretio, L. (2007). Un breve apunte histórico. Boletín Electrónico de Noticias de Educación a Distancia. 1-7. Extraído el 2 de junio, 2009 de http://www.uned.es/catedraunesco-ead/editorial/p7-6-2007.pdf

García-Valcárcel, A. (2003). Tecnología Educativa. Implicaciones educativas del desarrollo tecnológico. Madrid: La Muralla.

Gargallo López, B.; Suárez Rodríguez, J. y Almerich Cerveró, G. (2006). La influencia de las actitudes de los profesores en el uso de las nuevas tecnologías. Revista española de pedagogía, 233, 45-66.

Gil Flores, J. (1992). La metodología de investigación mediante grupos de discusión. Enseñanza, 10-11, 199-214.

Hammond, M.; Crosson, S.; Fragkouli, E.; Ingram, J.; Johnston-Wilder, P.; JohnstonWilder, S.; Kingston, Y.; Pope, M. \& Wray, D. (2009). Why Do Some Student Teachers Make Very Good Use of ICT? An Exploratory Case Study. Technology, Pedagogy and Education, 18 (1), 59-73.

Harrison, C.; Comber, C.; Fisher, T.; Haw, K.; Lewin, C.; Lunzer, E.; McFarlane, A.; Mavers, D.; Scrimshaw, P.; Somekh, B. \& Watling, R. (2002). ImpaCT2: The Impact of ICT on Pupil Learning and Attainment. Coventry: Becta. Extraído el 22 de octubre, 2009 de www.becta.org.uk/research.

Haydn, T.A. y Barton, R. (2007). Common Needs and Different Agendas: How Trainee Teachers Make Progress in Their Ability to Use ICT in Subject Teaching. Some Lessons from the UK. Computers \& Education, 49 (4), 1018-1036.

Hernández Sampieri R.; Fernández Collado C. y Baptista Lucio P. (2003). Metodología de la investigación. México: Mc Graw Hill.

ISTE (2002). Educational Computing and Technology Standards for Technology Facilitation. Technology Leadership and Secondary Computer Science Education. Extraído el 21 de septiembre, 2009 de http://www.iste.org.

Jonassen, D.H. (2006). Modeling with technology: Mindtools for conceptual change. Columbus, $\mathrm{OH}$ : Pearson-Prentice Hall.

Karsenti, T. (2004). Impact des TIC sur l'attitude, la motivation et le changement dans les pratiques pédagogiques des futurs enseignants. En M. Tardif y C. Lessard (dir.). La profession d'enseignant aujourd'hui: évolutions et perspectives internationals. Québec/Belgique: Presses de l'Université Laval/de Boeck.

Knezek, G. y Christensen, R. (2002). Impact of new information technologies on teachers and students. Education and Information Technologies, 7 (4), 369-376.

McFarlane, A. (2001). Perspectives on the relationships between ICT and assessment. Journal of Computer Assisted Learning, 17, 227-234.

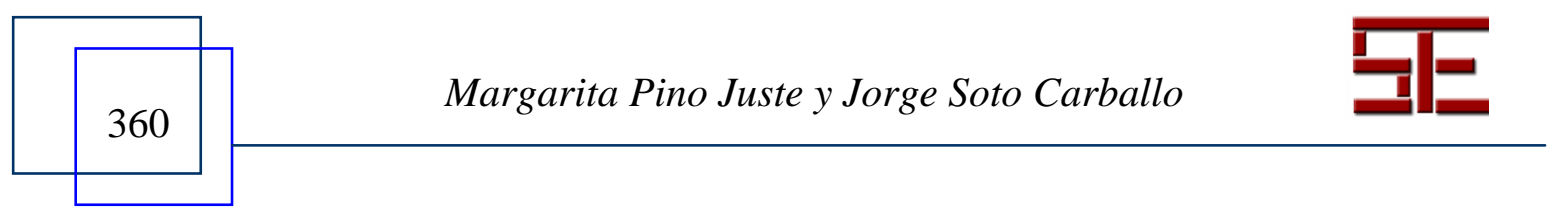




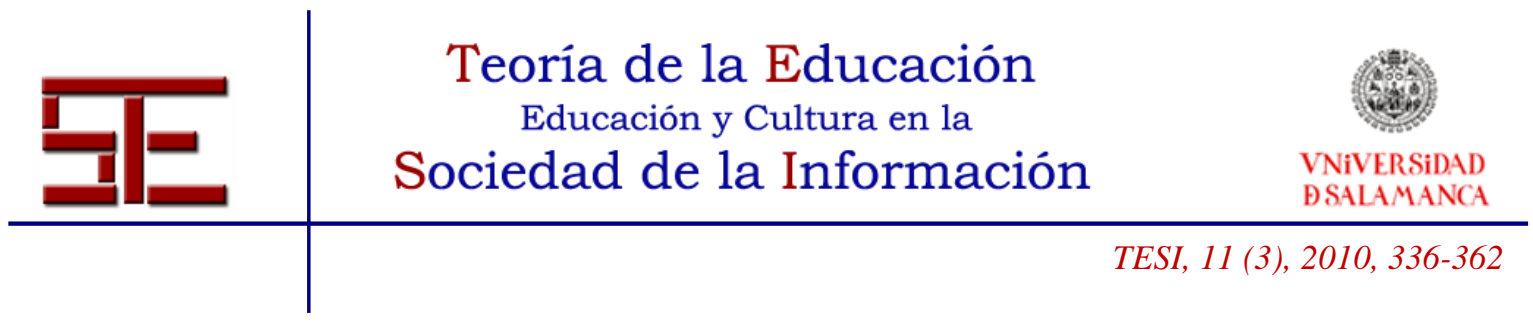

McMillanm, J. y Shumacher, S. (2005). Investigación Educativa: Una introducción conceptual. Madrid: Pearson.

Mérida Serrano, R (2006). Convergencia europea y la formación de competencias para la Educación Infantil en la Universidad. Revista de Educación, 341, 663-686.

Mominó, J.M. y Sigalés, C. (Coord) (2009). La integración de Internet en la educación escolar española. Situación actual y perspectivas de futuro. Barcelona: Instituto IN3 de la UOC-Universitat Oberta de Catalunya-Fundación telefónica.

Mooij, T. (2004). Optimising ICT effectiveness in instruction and learning: multilevel transformation theory and a pilot project in secondary education. Computers \& Education, 42, 25-44.

Mutton, T.; Mills, G.; McNicholl, J. (2006). Mentor Skills in a New Context: Working with Trainee Teachers to Develop the Use of Information and Communications Technology in Their Subject Teaching. Technology, Pedagogy and Education, 15 (3), 337-352.

Nichol J. \& Watson K. (2003). Editorial: rhetoric and reality - the present and future of ICT in education. British Journal of Educational Technology, 34, 1-6.

OECD (2001). Learning to change: ICT in schools. Paris: OECD.

Ottesen, E. (2006). Learning to Teach with Technology: Authoring Practised Identities. Technology, Pedagogy and Education, 15 (3), 275-290.

Prins, F.J.; Nadolski, R.J.; Berlanga, A.J.; Drachsler, H.; Hummel, H.G.K.; Koper, R. (2008). Competence Description for Personal Recommendations: The Importance of Identifying the Complexity of Learning and Performance Situations. Educational Technology \& Society, 11 (3), 141-152.

Reynolds D., Treharne D. \& Tripp H. (2003). ICT: the hopes and the reality. British Journal of Educational Technology, 34, 151-167.

Rosen, L. y Weil, M. (1995). Computer availability, computer experience and technophobia among public school teachers. Computers in Human Behavior, 11 (1), 931.

Scrimshaw, P. (2004). Enabling teachers to make successful use of ICT. Extraído el 7 de novimembre, 2009, de http://partners.becta.org.uk/uploaddir/downloads/page_documents/research/enablers.pdf Selwyn, N. (2003). Exploring patterns of computer use in schools. Welsh Journal of Education, 1, 75-97.

Selwyn, N. (1999). Why the computer is not dominating schools: A failure of policy or a failure of practice? Cambridge Journal of Education, 29 (1), 77-91.

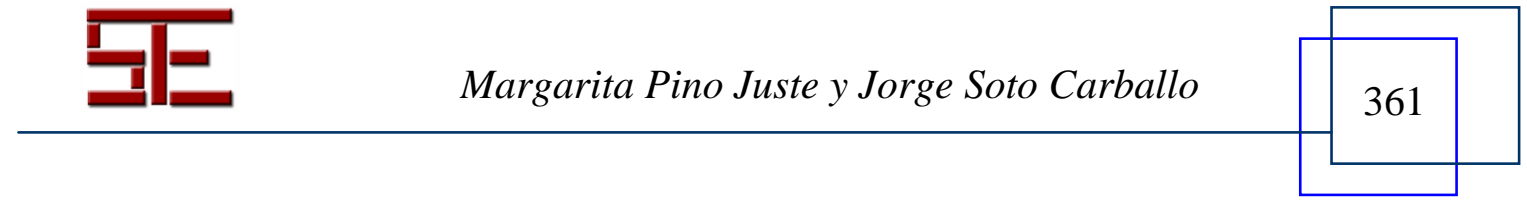




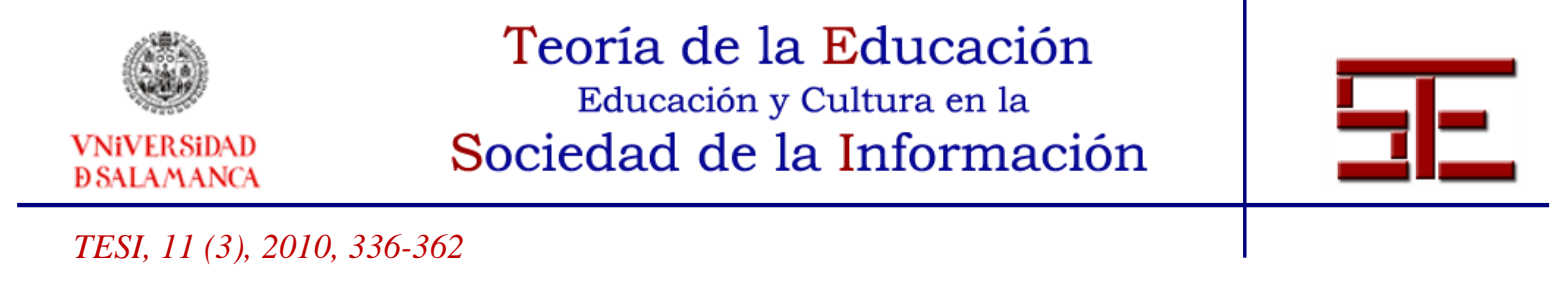

Strudler, N., McKinney, M., Jones, P. y Quinn, L. (1999). First-year teachers' use of technology: preparation, expectations and realities. Journal of Technology and Teacher Education, 7 (2), 115-129.

Tejedor Tejedor, F. J. y García-Valcarcel, A.M. (2006). Competencias de los profesores para el uso de las TIC en la enseñanza. Análisis de sus conocimientos y actitudes. Revista española de pedagogía, 233, 21-44.

Touriñan López, J. M. (2004). La educación electrónica: Un reto de la sociedad digital en la escuela. Revista española de pedagogía, 227, 31-58.

Urkijo, M. (Coord.) (2004). Investigación: Integración de la TIC en centros de ESO. Bilbao: ISEI-IVEI).

Ward, V. M.; Bertrand, J. T. y Brown, L. F. (1991). The comparability of focus group and survey results. Evaluation Revue, 15 (2), 266-283.

Watson, G. (1997). Pre-service teachers' views on their information technology education. Journal of Information Technology for Teacher Education, 6 (3), 255-269.

Windchitl, M. y Sahl, K. (2002). Tracing Teachers' Use of Technology in a Laptop Computer School: The Interplay of Teacher Beliefs, Social Dynamics, and Institutional Culture. American Educational Research Journal, 39 (1), 165-205.

Para citar el presente artículo puede utilizar la siguiente referencia:

Pino Juste, M. y Soto Carballo, J. (2010). Identificación del dominio de competencias digitales en el alumnado del grado de magisterio, en Orejudo González, J.P. (Coord.) Perspectiva educativa y cultural de "juego de rol". Revista Teoría de la Educación: Educación y Cultura en la Sociedad de la Información. Vol. 11, n 3 . Universidad de Salamanca, pp. 336-362 [Fecha de consulta: dd/mm/aaaa].

http://campus.usal.es/ revistas_trabajo/index.php/revistatesi/article/view/7466/7483 